\title{
How the Entrepreneurial Top Management Team Setup Influences Firm Performance and the Ability to Raise Capital: A Literature Review
}

\author{
Konstantin Maschke, Department of Strategic Leadership and Global Management, Technische Universität Berlin, Germany, \\ E-Mail: maschke.k@gmail.com \\ Dodo zu Knyphausen-Aufse $\beta$, Department of Strategic Leadership and Global Management, Technische Universität Berlin, Germany, \\ E-Mail:knyphausen@strategie.tu-berlin.de
}

\begin{abstract}
This paper reviews research findings on entrepreneurial top management teams within the last 20 years. It concentrates on team-based management factors and their influence on a new venture's growth and ability to raise capital. This paper integrates recent findings and provides an overview of the current state of research. Moreover, it contributes to the overall topic by proposing five clusters of major team-specific influences, derives determinants of success and failure, and reveals recommendations for further research.
\end{abstract}

JEL-classification: M13

Keywords: entrepreneurial top management teams, team composition, team demographics, team dynamics, team turnover, team experience, team leadership, literature review, performance, venture capital, IPO.

Manuscript received February 22, 2011, accepted by Peter Walgenbach (Management) December 13, 2011.

\section{Introduction}

Every second newly founded firm in the European Union does not survive its first five years (Schrör 2007). As proposed by Shrader and Siegel (2007) and Hambrick and Mason (1984), this disappointing result may be determined by the founders' and top managers' key attributes and characteristics, which both reflect values and cognitive foundations on which the new venture is built. As Finkelstein, Hambrick, and Cannella (2009: 3) wrote, "The small group of people at the top of an organization can dramatically affect organizational outcomes. Executives make big and small decisions. They shape the framework by which their organizations hire, mobilize, and inspire others to make decisions. They represent their organizations in dealings with external constituencies." While this may be true for big organizations, it may be even truer for smaller and newly founded firms where powerful actors may find it easier to imprint their values and knowledge on all aspects of the emerging organization (e.g., Ensley, Pearce, and Hmieleski 2006).

This paper reviews empirical research outcomes and focuses on characteristics of entrepreneurial top management teams (ETMTs) and their relationship to firm growth and the ability to raise capital. Within the observed top management team (TMT) research literature, several terms and definitions of a firm's TMT were introduced. Besides management team terms in general, such as top management team (e.g., Chaganti, Watts, Chaganti, and Zimmerman-Treichel 2008), upper echelon (e.g., Hambrick and Mason 1984), or dominant coalition (e.g., Hambrick and Mason 1984), entrepreneurship studies also use terms such as new venture top management team (e.g., Ensley, Pearson, and Amason 2002), new venture team (e.g., Busenitz, Fiet, and Moesel 2005), entrepreneurial team (e.g., Vissa 
Figure 1: Management team development process

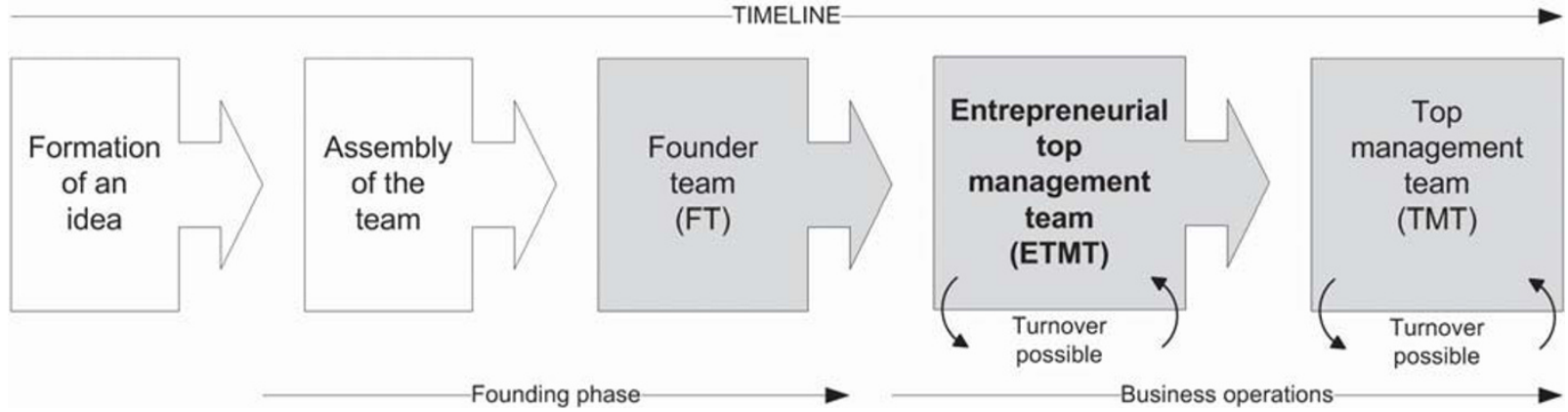

and Chacar 2009), founding team (e.g., Chaganti, Watts, Chaganti, and Zimmerman-Treichel 2008), start-up team (e.g., Franke, Gruber, Harhoff, and Henkel 2008), and early top management team (e.g., Beckman, Burton, and O’Reilly 2007). Differences in the definition range from the organizational levels included (e.g., executives, vice presidents, business unit heads), the necessity of an equity stake in the firm, active involvement in the strategic decision-making process, to the requirement of being a founding team (FT) member of the firm.

We define the term TMT for management teams in leading positions of an already-operating firm and with high involvement in the strategic decisionmaking process. The term FT is used instead for the group of people in the founding phase who decide to found a firm, develop the business model, purpose and plan, agree on the strategy, establish the funding of the firm, and provide the primary necessary capabilities to start the business. Here, differences arise in terms of intrinsic motivation, entrepreneurial vision, provided capabilities (Kor 2003), tacit knowledge about a venture's original purpose (Patzelt, zu Knyphausen-Aufseß, and Nikol 2008), stakes in the company, or team diversity and its impact on the process of founding versus the process of management. As a new term, we introduce ETMTs. Such teams are similar to TMTs, except for the additional constraint to serve in newly founded entrepreneurial firms and, therefore, lead to a more accurate definition of the teams considered in this paper. Due to the young age of entrepreneurial firms, a strong presence of founders in the ETMT is likely, but not necessarily required. Figure 1 provides an overview of the development path of teams in growing ventures to be applied in this paper. Due to a sound and careful selection of relevant sample papers, although a clear distinction is still necessary, the terms FT and TMT used in the papers can be expected to stand for the ETMT definition described above.

The assembly process of such teams is commonly based on individuals searching within personal social networks for partners to make an initial business idea happen and to supply a new venture with necessary resources (Kamm and Nurick 1993; Aldrich and Kim 2007). Several studies revealed results indicating a higher success rate of teamfounded ventures in contrast to individually founded ones (e.g., Cooper and Bruno 1977; Weinzimmer 1997). Drawing on human and social capital theory, teams can be assumed to have a great influence on a firm's development and thus can be seen as a crucial success factor and determinant of a new venture's performance. The advantages of entrepreneurial teams stem from the diversity and complementarity of skills, knowledge, characteristics, and experience (Chowdhury 2005). As entrepreneurial teams are responsible for many new venture foundings and the lonely heroic entrepreneur doing everything by him- or herself is more a myth than a real popular assembly (e.g., Cooney 2005), this review addresses the more common approach of teambased new venture management processes (Kamm, Shuman, Seeger, and Nurick 1990; Gerber 1995) and aims at answering the question of which ETMT characteristics and capabilities have proven to significantly influence corporate growth, venture capital (VC) funding, and initial public offerings (IPOs) of new ventures. Providing an answer to this research question is helpful since the most recent review article with a comparable focus dates back to 1990 (Kamm, Shuman, Seeger, and Nurick 1990) and an updated synthesis of ETMT research is appropriate due to the important role of entrepreneurial activities in modern economies and has been called for by academics such as Chaganti, Watts, Chaganti, and Zimmerman-Treichel (2008). 
There are three benefits of such an endeavor: First, for scholars and the scientific community this review offers an overview of the current state of research. It uncovers not yet discussed issues within the entrepreneurial and TMT literature stream where more profound research is necessary and reveals contradictions and open questions in past research where clarification is yet required. The integrated results are expected to give an overview of success and risk factors determining a new venture's outcomes. Second, it should provide an integration of academic results on which founders and entrepreneurs can base their team composition decisions (e.g., Kamm and Nurick 1993) and set up a team structure which matches the venture's external requirements and strategic aims. Third, VCs can make use of discovered relationships and include research results into their own rating and validation scheme of new ventures and their ETMTs. Furthermore, angel investors and those responsible for selecting and composing new venture teams can derive guidelines on how to set up effective ETMTs for certain start-up ventures.

Our review started by introducing specific terminology and team definitions primarily used in ETMT research and continues with an explanation of human and social capital theories and organizational outcomes in an entrepreneurial context. We then describe the sampling and coding process of the reviewed sample comprising of 28 papers. The findings of our review procedure are discussed and integrated by distinguishing five clusters categorizing the independent variables. Significant relationships are then integrated in summary tables showing relevant identified ETMT factors influencing a new venture's growth and funding. A discussion of the results, a collection of influencing success and risk factors of ETMTs derived from the discussion, and a recommendation for future research avenues conclude this paper.

\section{Subject Matter and Underlying Theories}

This section covers the reasoning to include and exclude certain dependent variables to this review and digs deeper into the main theories applied by the underlying sample papers.

\subsection{Growth and Fundraising}

This review focuses on two major firm outcomes as indicators of entrepreneurial success - a new ven- ture's performance in terms of growth and the ability to raise capital via external funding or IPOs to facilitate a firm's growth path and to achieve strategic goals (e.g., Delarue, van Hootegem, Procter, and Burridge 2008). As the profitability of especially young firms in the start-up phase has only limited informative value, such measures were not included. Neglecting motivations such as selffulfillment and an existential necessity as well as diverse non-financial and operating indicators for a firm's success such as the creation of jobs, gain of market shares, environmental protection, or foreign development aid, we focus on the economic yields of entrepreneurial founding and are interested in analyzing how diverse factors influence such success indicators.

As different performance measures can reveal different findings, scholars often used several measures to account for different dimensions (e.g., Sapienza and Grimm 1997; Amason, Shrader, and Tompson 2006). Sales growth is clearly the most consistently applied new venture performance indicator in prior research. This is a critical factor and primary objective of new ventures in the stage of commercialization and growth. It indicates the utilization of economies of scale, the ability to resist environmental disruptions, and the increase in power (e.g., Vissa and Chacar 2009; Eisenhardt and Schoonhoven 1990; Ensley, Pearson, and Amason 2002). Besides sales growth, employee growth and asset growth are frequently used in research studies (e.g., Chaganti, Watts, Chaganti, and ZimmermanTreichel 2008; Colombo and Grilli 2005; Patzelt, zu Knyphausen-Aufseß, and Nikol 2008). Beckman, Burton, and O'Reilly (2007) add another two indicators of entrepreneurial success: the ability to attract external funding and the ability to successfully complete an IPO. As new ventures often need to obtain financing from outside investors due to internal financial limitations, $\mathrm{VC}$ and private equity (PE) funding and going public are the most common and most important milestones and sources for entrepreneurial firms to receive external funds (Beckman, Burton, and O’Reilly 2007; Hsu 2007).

\subsection{Underlying Theories and Concepts}

The majority of the published articles in the ETMT research stream draw on a set of recurring theories and concepts. Such theories give a theoretical answer to the question of why particular independent variables are expected to have an influence on de- 
pendent variables and thus were tested within a research study. For example, upper echelon theory assumes individual characteristics of the top management of a firm to lead to certain interpretations of internal and external situations, which is the foundation of strategic decision-making processes and ultimately influences firm performance (Hambrick and Mason 1984; Carpenter, Geletkanycz, and Sanders 2004). Instead, the resource-based view expects the application of all valuable, rare, difficultto-imitate, and non-substitutable resources available to a firm to be a source of competitive advantages (Wernerfelt 1984; Barney 1991). Signaling theory is primarily used in studies describing the interaction between ETMTs and investors, as it explains how to overcome asymmetric information by conveying credible signals and information from one party to another (Spence 1973; Connelly, Certo, Ireland, and Reutzel 2011). However, the primarily applied concepts to models and studies in ETMT research are the theories of human and social capital. They will be outlined in more detail below.

The theory of human capital perceives labor as heterogeneous and explains differences in an individual's productivity and wages through the disparity of human capital such as capabilities, skills, knowledge, competences, and abilities (Shrader and Siegel 2007; Becker 1964). Such human capital is gained via training, work experience, education, and accumulated skills and knowledge (Becker 1964). Enhanced productivity, certain managerial characteristics, and experience of a TMT can lead to competitive advantages, increased firm performance, and to the ability to make more informed strategic decisions (Shrader and Siegel 2007; Gimeno, Folta, Cooper, and Woo 1997). Colombo and Grilli (2005), for example, assumed a close relationship between the knowledge and skills of founders and the distinctive capabilities of a new venture. Training and wages for experienced and skilled managers can be seen as a firm's investment in human capital, expecting to benefit from higher productivity and added economic value (Becker 1964). From the perspective of the employee, wages are the returns from prior investments into one's own human capital (Shrader and Siegel 2007).

Especially entrepreneurial firms are determined by the quality of their FT, TMT, and employees. Individual capabilities of human capital are widely proxied by education, academic degrees, prior work or industry experience, received training, and in the context of TMTs by team heterogeneity and size, as the number of team members correlates with experience (e.g., Hsu 2007; Eisenhardt and Schoonhoven 1990; Baum and Silverman 2004). Becker (1964) distinguished between general (acquired via general professional experience and education; similar productivity effect for all firms) and special (acquired via industry or firm-specific experience; only specifically applicable to a firm) human capital. Besides the capability effect that is commonly associated with human capital, the financial abilities of an individual known as wealth effect matter especially in an entrepreneurial context (Shrader and Siegel 2007). "Firms that are established by wealthier individuals are less affected by financial constraints as greater personal capital is available to finance firms' operations" (Colombo and Grilli 2005: 796). Moreover, investment of personal wealth signals strong commitment and credibility to potential VC investors.

However, human capital theory is not without criticism among the scientific community. Some researchers stated that the earned wage is not only determined by an individual's human capital, but also by personal character, socialization within the firm, relations to insiders (e.g., friends, family), gender, or discrimination (e.g., Sweetland 1996). Others such as Block (1990) complained about the phrase "capital" and prefer to define human capabilities as a commodity and independent social force.

While human capital emphasizes what one knows and what one has experienced, Coleman (1988), Putnam, Leonardi, and Nanetti (1994), and Burt (1995) proposed that social capital instead is about who one knows and which social ties one can resort to. Social capital available to a firm refers to benefits from its members' collective networks, social relations, and underlying social structures (Hsu 2007; Adler and Kwon 2002; Witt 2004; West 2007), which can, e.g., stem from prior involvement in an entrepreneurial community (Adler and Kwon 2002; Hsu 2007), professional experience, or friendships. Social capital is a result of dynamic interaction and is created and developed by the ETMT members' social activities (Sahaym 2005). Such social capital consists of trust, sympathy, and forgiveness offered by social ties to individuals. These resources and capabilities are embedded within social systems and create a collective cognition (Sahaym 2005; West 2007). It is seen as a valuable intangible asset as it 
provides access to broader and more qualified information and resources, a person's goodwill, and can facilitate individuals' or firms' action and goal achievement, and increase influence and power (Adler and Kwon 2002). Potential disadvantages are considerable investments in social capital with unpredictable outcomes, possible fragmentation, and separation of teams with high solidarity and sympathy from the broader whole, or negative effects of power which lead to dependencies of the focal actor (Adler and Kwon 2002).

"Strong social ties based on personal relationships often play important economic and social roles during new venture creation" (Sahaym 2005: 2). External ties to individuals or organizations, also known as "bridging", can be gathered and enhanced by a solid career history or industry experience. Internal ties to colleagues or team members, also known as "bonding", facilitate cooperative relationships and team cohesion and reduce conflict (Beckman, Burton, and O'Reilly 2007; Adler and Kwon 2002). Both bridging and bonding are important requirements for entrepreneurs to attract VCs and improve a new venture's performance by leveraging social ties (Vissa and Chacar 2009; Beckman, Burton, and O’Reilly 2007; Witt 2004). Social capital is not to be seen as a static fabric, but much more as a highly dynamic network with changing ties and nodes (Hsu 2007). Adler and Kwon (2002) described three influencing factors facilitating the development of social capital: opportunities offered by external and internal ties which allow leveraging resources and acting together; intrinsic motivation engendered through deeply internalized norms and socialization to define collective goals and enact together as a team; and the abilities of individuals which can enrich or support other actors' target achievement. Typical proxies can be the span and range of an individual's social network, internal team cohesion, the ability of an ETMT to self-recruit its employees, or the ability to raise $\mathrm{VC}$ funds via personal social ties.

Social capital theory suffers from a great deal of criticism for being poorly defined and conceptualized due to its frequent application in diverse research fields. This problem stems from its multidimensional characteristic and authors such as Liu and Besser (2003) and Woolcock and Narayan (2000) tried to integrate these dimensions into a structure.
The introduced theories are commonly applied when researchers tend to explain phenomena within ETMT research. The sample of this literature review, which is identified and coded in the following chapter, resorts to such theories by deriving hypotheses and testing them with statistical models. Frequent application of these theories leads to increasing evidence of their validity in ETMT research. Thus, especially human and social capital theories experienced such intense attention in prior studies and have proven to explain ETMT impact comparably well.

\section{Methodology}

The extracted and synthesized data considered in this review followed a structured process from the data collection and evaluation to the analysis and interpretation via a sampling and coding procedure.

\subsection{The Sampling Process}

The sampling process was conducted via a multistage selection and filter procedure. Primary requirements for papers being included in the literature review sample were the matching of certain keywords in the title or abstract, publication in highquality journals, quantitative methods and models, empirical content, as well as ensuring substantive and empirical relevance for the focus of this paper. Since the included papers are a representative sample in the sense of Cooper (1988), we claim to aptly give a reasonable overview of research and results between 1989 and 2009. As the databases searched and the journals considered are of high quality and have high recognition in the scientific community (e.g., Tacheva 2007; Ennen and Richter 2010), the extracted results are expected to be of high conceptual and methodological correctness. The comparison and integration of quantitative research outcomes are of primary interest. The sampling process was conducted following Newbert (2007); details are explained in Appendix A: Sampling process.

This process revealed a sample of 28 quantitative papers, all highly relevant, suitable, and representative for the focus of this literature review. This sample is limited to papers dealing with relevant dependent variables (venture growth and funding) and TMTs in an entrepreneurial context. As Figure 2 shows, there has been growing interest in entrepreneurial and TMT research over the last 20 years. 
Figure 2: Distribution of sample papers

Distribution of the sample papers by publishing date ( $\Sigma 28$ paper)
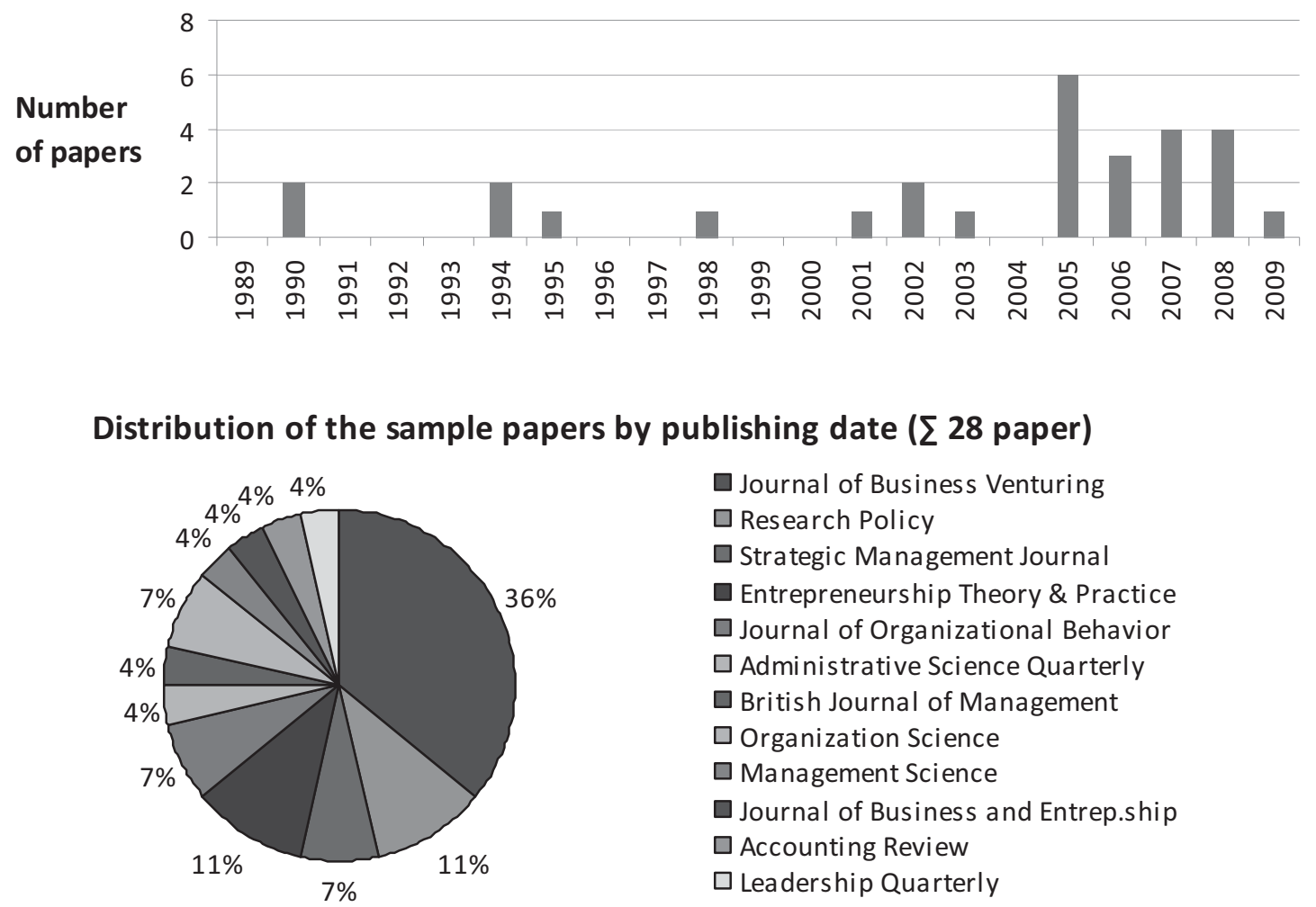

Distribution of the sample papers by dependent variable ( $\Sigma 28$ paper)

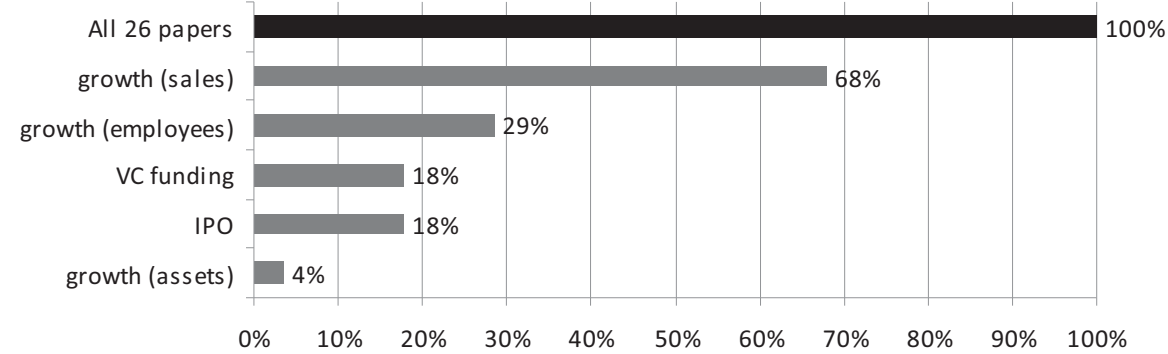

Not surprisingly, more than two thirds of the sample papers were published in journals with a strong focus on management, strategy, and venturing. In terms of the dependent variables (often more than one dependent variable per paper), there is a clear emphasis on sales growth ( $68 \%$ of the sample papers) and employee growth (29\%) figures in the developed models of the sample. VC funding (18\%),
IPOs (18\%), and asset growth (4\%) are examined less frequently. No papers in the relevant context emerged before 1990, then minor research activities took place until 2004, and from then on, there has been growing interest up to the present today. In recent years relevant research activities seem to drop and a certain level of saturation seems to have been reached. This evidences and supports our 
thinking that it is the right time for a literature review integrating and synthesizing results from twenty years of research, providing the scientific community with an overview of findings and yet-tobe explored issues for future research. Another 68 papers which deal with the same topic and are worth to mention were identified and summarized in Appendix B: Auxiliary sample. These papers contribute to the understanding of the research stream but did not survive the sampling procedure. Moreover, the articles of Brettel (2002), Franke, Gruber, Henkel, and Hoisl (2004), Schmelter (2004), and Chlosta and Kissel (2011) with comparably high thematically overlap in German were found during the sampling process but likewise did not survive the complete procedure. Articles in languages other than English or German have not been included in our sampling process.

\subsection{The Coding Process}

Thorough and careful reading of the papers of the sample was accompanied by aggregating the essential information and results in a separated coding template which serves as a coding book, as suggested by Randolph (2009) for each paper. The process followed a three-step approach for every sample paper, which is determined by the focus of this literature review on research outcomes and integration: First, a paper summary model was developed containing all relevant information regarding macro data (e.g., publishing year, journal, authors), paper details and categorization (e.g., hypotheses, major topics, used theories), quantitative model and results (e.g., variables, significant relationships, applied methodologies), data collection procedure (e.g., data source, sample size), as well as terminology and definitions. Second, a coding model was derived focusing on data relevant for integrating and comparing results and approaches among the papers of the sample. Third, a structure model was derived to explore major categorization options in order to set up a reasonable, sound, and clear structure for the literature review to follow.

\section{Review of the Current State of Research}

The in-depth analysis of the 28 sample papers is structured into five sections dealing with teamspecific factors and one final section aggregating the major findings of the sample in summary tables.
The sections were derived during the coding process from clusters of the structure model, each covering a distinct defined set of linked variables, namely, demographics, leadership, turnover, dynamics, and experience. Each section is separated into subsections of different major independent variables and introduces theoretical expectations and previous findings of related research fields along with a description of the results in the sample. Table 1 gives an overview of all papers in the sample describing all independent, dependent, control, and interaction variables.

\subsection{Influence of Team Demographics}

As observable managerial characteristics are easy to collect and their influence on diverse firm factors is proven, the high share of papers including these variables in their models is not a big surprise.

The size of the team is an impact factor that is not very commonly tested, in contrast to a quite frequent use as a control variable. It is argued that large teams bring along high levels of affective conflict, unclear task definitions in an early firm's stage, and negative effect on social integration and relationship building (Amason and Sapienza 1997; Smith, Smith, Sims, O’Bannon, Scully, and Olian 1994). On the other hand, large teams are known for greater access to resources, greater ability to process, gather and absorb information, a larger bandwidth of specialization and diversity, and the ability to execute more tasks parallel (e.g., Salancik and Pfeffer 1978; Eisenhardt and Schoonhoven 1990). Eisenhardt and Schoonhoven (1990) and Cooper, Gimeno-Gascon, and Woo (1994) showed positive influence of team size on new venture sales and employee growth. Moreover, the teams' cumulative social capital and range of networks supports venture investments and leads to a higher probability of receiving funds aided by direct and indirect social ties to external investors (Shane and Stuart 2002).

Average team age is seen as a proxy for experience (e.g., industry, functional), but also decreased flexibility, increased resistance to status quo changes, and reduced risk-taking (Wiersema and Bantel 1992; Carlsson and Karlsson 1970). Instead, younger teams tend to take greater risks, pursue novel approaches, and have a higher affinity to rising markets and new technologies (Hambrick and Mason 1984). Results from Chaganti, Watts, Chaganti, and Zimmerman-Treichel (2008) of average team age and ethnic immigrants' presence on 
diverse firm growth variables (sales, assets, employees) revealed a clear picture: old teams not only reduce the positive relative effect of ethnic immigrants' presence on growth variables, but they are also associated with lower growth rates in general. Regarding minority groups, a positive effect of such in the ETMT on employee growth was confirmed by Cooper, Gimeno-Gascon, and Woo (1994). The same applies to male-dominated ETMTs.

Measures for ETMT's education are often used as proxies for organizational or human capital in the firm. Education in general is associated with a high probability of venture survival, greater growth rates, and venture performance (e.g., Almus and Nerlinger 1999; Cooper, Gimeno-Gascon, and Woo 1994; Stuart and Abetti 1990). An MBA degree, for example, reflects general managerial training of the respective member within the team.

Colombo and Grilli (2005) tested for the impact of education on employee growth and PE financing, coming to the conclusion that "[t]he likelihood of a firm resorting to outside private equity financing is found to increase with education attainments of founders [...]" (p. 809), measured via managerial and technical education of founders. Moreover, managerial graduate education, and to a lesser extent technical graduate education, both have a positive effect on employee growth. This is in line with findings from Cooper, Gimeno-Gascon, and Woo (1994) who confirmed a strong positive relationship between higher education and employee growth.

Summarizing, there is substantial agreement among the reviewed studies concerning a strong relationship between ETMT education and new venture growth. Although, Stuart and Abetti (1990) showed findings with a negative influence of ETMT education on a venture growth index, as the underlying sample was "quite well educated with all having at least some college, 15 had master's degrees and 7 had PhDs" (p. 159), an effect of "overeducation" might have influenced team efficiency negatively. For example, the existence of a $\mathrm{PhD}$ degree holder in the team was found by Hsu (2007) to be negatively associated with a VC investor to be a direct contact of a team member. "[...] $\mathrm{PhD}$ recipients may not have invested in activities to enhancing their social capital in entrepreneurial [...] communities [...]" (Hsu 2007: 736).

The number of firm founders who are still members of the ETMT indicates the influence and existence of the original business model and historical business premises (Kor 2003). Due to their knowledge of the initial business concept and its resources and capabilities at foundation, founders have the cognitive abilities to allocate limited resources to most-promising projects and rising opportunities (Kor and Mahoney 2000; Barringer, Jones, and Neubaum 2005).

In line with this argumentation, Kor (2003) found a clear positive relationship between existing founders in the ETMT and a firm's sales growth. Interactions show that this relationship weakens as the level of shared team acquaintance and experience increases, due to potentially emerging conflicts stemming from authority-claiming within the team. The same effect holds when industry-specific management experience in the team increases. However, these negative influences are still overcompensated by the strong positive influence of founders existing in the team. However, founders tend to delay critical decisions regarding corrections of courses in which they have invested substantial resources or are responsible for failure (Patzelt, zu KnyphausenAufseß, and Nikol 2008). In order to professionalize a firm's management, external investors might force founders to give up leading management positions in the firm and replace them with external managers (Hellmann and Puri 2002).

The cognitive comprehensiveness of a team describes the ability for complex and innovative decision-making. The impact of cognitive comprehensiveness as a team process variable on a firm providing a collective understanding of a venture's success drivers, effective relationships among team members, and efficient interaction processes is of course expected to be high (e.g., Chowdhury 2005; Miller, Burke, and Glick 1998). This assumption has already found evidence in Simons, Pelled, and Smith (1999) with positive effects on sales growth. Chowdhury (2005) confirmed a positive relationship between a team's cognitive comprehensiveness and team effectiveness which is also expected to influence sales growth positively.

More than one third of the sample papers deal with team heterogeneity and its influence on the firm. As heterogeneity among the team provides a broader perspective, more experience, knowledge, skills, abilities, insight in decision-making (Chaganti, Watts, Chaganti, and Zimmerman-Treichel 2008), and leaves room for constructive conflict, benefits for the firm and organizational outcomes can be assumed. Moreover, diversity widens the network 


\section{Table 1: Overview of the sample papers}

\begin{tabular}{|c|c|c|c|c|c|c|}
\hline Title & Author(s) & Year & Independent variables & Dependent variables & Control variables & Interaction variables \\
\hline $\begin{array}{l}\text { Leveraging ties: the } \\
\text { contingent value of } \\
\text { entrepreneurial } \\
\text { teams' external } \\
\text { advice networks on } \\
\text { Indian software } \\
\text { venture perform- } \\
\text { ance }\end{array}$ & $\begin{array}{l}\text { Vissa, Balagopal; } \\
\text { Chacar, Aya S. }\end{array}$ & 2009 & $\begin{array}{l}\text { - TMT shared strategic cognition and } \\
\text { consensus } \\
\text { - TMT cohesion } \\
\text { - TMT heterogeneity: functional back- } \\
\text { ground } \\
\text { - TMT network constraint }\end{array}$ & - sales growth & $\begin{array}{l}\text { - number of employees }(\log ) \\
\text { - venture age } \\
\text { - PE/VC funding received } \\
\text { - TMT funding experience }\end{array}$ & $\begin{array}{l}\text { - TMT network constraint x TMT shared strategic } \\
\text { cognition and consensus } \\
\text { - TMT network constraint x TMT cohesion }\end{array}$ \\
\hline $\begin{array}{l}\text { Ethnic-immigrants } \\
\text { in founding teams: } \\
\text { effects on prospec- } \\
\text { tor strategy and } \\
\text { performance in new } \\
\text { internet ventures }\end{array}$ & $\begin{array}{l}\text { Chaganti, Ra- } \\
\text { jeswararaoS; } \\
\text { Watts, Allison } \\
\text { D.; Chaganti, } \\
\text { Radha; Zim- } \\
\text { merman- } \\
\text { Treichel, Monica }\end{array}$ & 2008 & $\begin{array}{l}\text { - TMT presence of ethnic immigrants } \\
\text { - TMT size } \\
\text { - TMT avg. age }\end{array}$ & $\begin{array}{l}\text { - sales growth } \\
\text { - asset growth } \\
\text { - employee growth } \\
\text { - prospector strategy } \\
\text { (focus on R\&D and } \\
\text { marketing) }\end{array}$ & - & $\begin{array}{l}\text { - TMT presence of ethnic immigrants x TMT size } \\
\text { - TMT presence of ethnic immigrants x TMT avg. age }\end{array}$ \\
\hline $\begin{array}{l}\text { Top management } \\
\text { teams, business } \\
\text { models, and per- } \\
\text { formance of bio- } \\
\text { technology ven- } \\
\text { tures: an upper } \\
\text { echelon perspective }\end{array}$ & $\begin{array}{l}\text { Patzelt, Holger; } \\
\text { zu Knyphausen- } \\
\text { Aufseß, Dodo; } \\
\text { Nikol, Petra }\end{array}$ & 2008 & $\begin{array}{l}\text { - TMT university graduates } \\
\text { - founders in TMT } \\
\text { - TMT biotech industry experience } \\
\text { - TMT pharma industry experience } \\
\text { - business model }\end{array}$ & - employee growth & $\begin{array}{l}\text { - number of employees } \\
\text { - venture age }\end{array}$ & $\begin{array}{l}\text { - TMT university graduates } x \text { business model } \\
\text { - founders in TMT x business model } \\
\text { - TMT biotech industry experience x business model } \\
\text { - TMT pharma industry experience } x \text { business model }\end{array}$ \\
\hline $\begin{array}{l}\text { The influence of top } \\
\text { management team } \\
\text { heterogeneity on the } \\
\text { capital raised } \\
\text { through an initial } \\
\text { public offering }\end{array}$ & $\begin{array}{l}\text { Zimmerman, } \\
\text { Monica A. }\end{array}$ & 2008 & $\begin{array}{l}\text { - TMT heterogeneity: functional back- } \\
\text { ground } \\
\text { - TMT heterogeneity: educational } \\
\text { specialization } \\
\text { - TMT heterogeneity: age } \\
\text { - TMT heterogeneity: tenure }\end{array}$ & $\begin{array}{l}\text { - IPO value (capital } \\
\text { raised at IPO) }\end{array}$ & $\begin{array}{l}\text { - Year of IPO } \\
\text { - Hot market } \\
\text { - Firm age } \\
\text { - TMT size } \\
\text { - Equity raised } \\
\text { - Prior sales } \\
\text { - Team tenure } \\
\text { - Underwriter reputation } \\
\text { - VC backing } \\
\text { - Entrepreneurial experience } \\
\text { - Founder experience }\end{array}$ & - \\
\hline
\end{tabular}


Table 1 continued: Overview of the sample papers

\begin{tabular}{|c|c|c|c|c|c|c|}
\hline Title & Author(s) & Year & Independent variables & Dependent variables & Control variables & Interaction variables \\
\hline $\begin{array}{l}\text { Founding the fu- } \\
\text { ture: path depend- } \\
\text { ence in the evolu- } \\
\text { tion of top man- } \\
\text { agement teams from } \\
\text { founding to IPO }\end{array}$ & $\begin{array}{l}\text { Beckman, Chris- } \\
\text { tine M.; Burton, } \\
\text { M. Diane }\end{array}$ & 2008 & $\begin{array}{l}\text { - TMT functional experience } \\
\text { - TMT functional structure }\end{array}$ & $\begin{array}{l}\text { - time to receive VC } \\
\text { - time to IPO }\end{array}$ & $\begin{array}{l}\text { - firm size } \\
\text { - } \text { medical-related industry } \\
\text { - } \text { innovation strategy } \\
\text { - } \text { marketing strategy } \\
\text { - number of VC deals } \\
\text { - } \text { cumber of IPOs in industive VC } \\
\text { - Avg. team size } \\
\text { - Product developed } \\
\text { - Share of founders on TMT } \\
\text { - Cumulative TMT entrances } \\
\text { - Cumulative TMT exits }\end{array}$ & $\begin{array}{l}\text { - TMT functional experience x TMT functional struc- } \\
\text { ture }\end{array}$ \\
\hline $\begin{array}{l}\text { Experienced entre- } \\
\text { preneurial founders, } \\
\text { organizational } \\
\text { capital, and venture } \\
\text { capital funding }\end{array}$ & Hsu, David H. & 2007 & $\begin{array}{l}\text { - high recruiting via FT network } \\
\text { - TMT founding experience (log) } \\
\text { - TMT with MBA degree } \\
\text { - TMT with PhD degree } \\
\text { - high prior start-up return }\end{array}$ & $\begin{array}{l}\text { - VC funding through } \\
\text { direct personal tie of a } \\
\text { founding team mem- } \\
\text { ber } \\
\text { - valuation of start-up } \\
\text { before VC funding }\end{array}$ & $\begin{array}{l}\text { - FT size (log) } \\
\text { - venture age (log) } \\
\text { - number of employees after } \\
\text { VC funding (log) } \\
\text { - angel investor prior to VC } \\
\text { - } \text { nunding } \\
\text { - } \text { multiple financing offers } \\
\text { received } \\
\text { - equity taken out after VC } \\
\text { - } \text { sunding (log) } \\
\text { - strategy: technology-based } \\
\text { - strategy: product-based } \\
\text { - industry: internet } \\
\text { - industry: software } \\
\text { - industry: biotechnology } \\
\text { - industry: communications } \\
\text { - year of VC funding }\end{array}$ & $\begin{array}{l}\text { - industry: internet x TMT with MBA degree } \\
\text { - industry: internet x TMT with PhD degree } \\
\text { - industry: internet x high recruiting via FT network }\end{array}$ \\
\hline
\end{tabular}




\section{3}

BuR - Business Research

Official Open Access Journal of VHB

ess Research (VHB)

Volume 5 | Issue 1 | May 2012 | 83-123

Table 1 continued: Overview of the sample papers

\begin{tabular}{|c|c|c|c|c|c|c|}
\hline Title & Author(s) & Year & Independent variables & Dependent variables & Control variables & Interaction variables \\
\hline $\begin{array}{l}\text { A contextual exami- } \\
\text { nation of new ven- } \\
\text { ture performance: } \\
\text { entrepreneur lead- } \\
\text { ership behavior, top } \\
\text { management team } \\
\text { heterogeneity, and } \\
\text { environmental } \\
\text { dynamism }\end{array}$ & $\begin{array}{l}\text { Hmieleski, Keith } \\
\text { M.; Ensley, } \\
\text { Michael D. }\end{array}$ & 2007 & $\begin{array}{l}\text { - environmental dynamism } \\
\text { - TMT heterogeneity } \\
\text { - leadership: directive } \\
\text { - leadership: empowering }\end{array}$ & $\begin{array}{l}\text { - index of revenue } \\
\text { growth and employee } \\
\text { growth }\end{array}$ & $\begin{array}{ll}\text { - } & \text { venture age } \\
\text { - } & \text { sales (log) } \\
\text { - } & \text { TMT size } \\
\text { - } & \text { number of employees }(\log )\end{array}$ & $\begin{array}{l}\text { - environmental dynamism x TMT heterogeneity } \\
\text { - environmental dynamism x leadership: directive } \\
\text { - environmental dynamism x leadership: empowering } \\
\text { - TMT heterogeneity x leadership: directive } \\
\text { - TMT heterogeneity x leadership: empowering } \\
\text { - TMT heterogeneity x environmental dynamism x } \\
\text { leadership: directive } \\
\text { - TMT heterogeneity x environmental dynamism x } \\
\quad \text { leadership: empowering }\end{array}$ \\
\hline $\begin{array}{l}\text { Early teams: The } \\
\text { impact of team } \\
\text { demography on VC } \\
\text { financing and going } \\
\text { public }\end{array}$ & $\begin{array}{l}\text { Beckman, Chris- } \\
\text { tine M.; Burton, } \\
\text { M. Diane; } \\
\text { O'Reilly, Charles }\end{array}$ & 2007 & $\begin{array}{l}\text { - FT heterogeneity: functional back- } \\
\text { ground } \\
\text { - TMT heterogeneity: functional back- } \\
\text { ground } \\
\text { - TMT founding experience } \\
\text { - TMT management experience } \\
\text { - FT founding experience } \\
\text { - FT management experience } \\
\text { - TMT additions } \\
\text { - TMT departures } \\
\text { - FT departures } \\
\text { - FT work experience: no. prior compa- } \\
\text { nies } \\
\text { - FT work experience: no. overlapping } \\
\text { prior companies } \\
\text { - TMT work experience: no. prior } \\
\text { companies } \\
\text { - TMT work experience: no. overlap- } \\
\text { ping prior companies }\end{array}$ & $\begin{array}{l}\text { - VC funding } \\
\text { - going public (IPO) }\end{array}$ & $\begin{array}{l}\text { - number of employees } \\
\text { - venture industry } \\
\text { - PE/VC financing received } \\
\text { - industry: annual no. of IPOs } \\
\text { - industry: annual VC funding } \\
\text { volume } \\
\text { - TMT avg. prior positions } \\
\text { - FT size } \\
\text { - TMT size } \\
\text { - TMT mean tenure } \\
\text { - TMT tenure heterogeneity }\end{array}$ & - \\
\hline
\end{tabular}




\section{3}

BuR - Business Research

Official Open Access Journal of VHB

German Academic Association for Business Research (VHB)

Volume 5 | Issue 1 | May 2012 | 83-123

Table 1 continued: Overview of the sample papers

\begin{tabular}{|c|c|c|c|c|c|c|}
\hline Title & Author(s) & Year & Independent variables & Dependent variables & Control variables & Interaction variables \\
\hline $\begin{array}{l}\text { Assessing the rela- } \\
\text { tionship between } \\
\text { human capital and } \\
\text { firm performance: } \\
\text { evidence from } \\
\text { technology-based } \\
\text { new ventures }\end{array}$ & $\begin{array}{l}\text { Shrader, Rod; } \\
\text { Siegel, Donald S. }\end{array}$ & 2007 & $\begin{array}{l}\text { - strategic breadth } \\
\text { - strategic aggressiveness } \\
\text { - strategic international intensity } \\
\text { - strategy: low cost } \\
\text { - strategy: differentiation } \\
\text { - strategy: focus } \\
\text { - TMT industry experience } \\
\text { - TMT technical experience } \\
\text { - TMT marketing experience } \\
\text { - TMT finance experience } \\
\text { - TMT international experience } \\
\text { - TMT founding experience }\end{array}$ & $\begin{array}{l}\text { - sales growth } \\
\text { - profitability (average } \\
\text { of ROI, ROS and ROA) }\end{array}$ & $\begin{array}{l}\text { - venture age } \\
\text { - industry: growth } \\
\text { - industry: global integration } \\
\text { - industry: technological in- } \\
\text { tensity } \\
\text { - industry: competitive inten- } \\
\text { sity }\end{array}$ & $\begin{array}{l}\text { - } \text { strategic breadth x TMT founding experience } \\
\text { - } \text { strategic aggressiveness x TMT industry experience } \\
\text { - strategic aggressiveness x TMT technical experience } \\
\text { - experience } \\
\text { - strategy: low cost x TMT industry experience } \\
\text { - strategy: differentiation x TMT marketing experience } \\
\text { strategy: differentiation x TMT technical experience }\end{array}$ \\
\hline $\begin{array}{l}\text { The importance of } \\
\text { vertical and shared } \\
\text { leadership within } \\
\text { new venture top } \\
\text { management teams: } \\
\text { Implications for the } \\
\text { performance of } \\
\text { startups }\end{array}$ & $\begin{array}{l}\text { Ensley, Michael } \\
\text { D.; Hmieleski, } \\
\text { Keith M.; Pearce, } \\
\text { Craig L. }\end{array}$ & 2006 & $\begin{array}{l}\text { - Vertical directive leadership } \\
\text { - Vertical transactional leadership } \\
\text { - Vertical transformational leadership } \\
\text { - Vertical empowering leadership } \\
\text { - Shared directive leadership } \\
\text { - Shared transactional leadership } \\
\text { - Shared transformational leadership } \\
\text { - Shared empowering leadership }\end{array}$ & $\begin{array}{l}\text { - index of revenue } \\
\text { growth and employee } \\
\text { growth }\end{array}$ & $\begin{array}{l}\text { - Firm age } \\
\text { - Number of employees } \\
\text { - TMT size }\end{array}$ & - \\
\hline
\end{tabular}


Table 1 continued: Overview of the sample papers

\begin{tabular}{|c|c|c|c|c|c|c|}
\hline Title & Author(s) & Year & Independent variables & Dependent variables & Control variables & Interaction variables \\
\hline $\begin{array}{l}\text { Newness and nov- } \\
\text { elty: Relating top } \\
\text { management team } \\
\text { composition to new } \\
\text { venture perform- } \\
\text { ance }\end{array}$ & $\begin{array}{l}\text { Amason, Allen } \\
\text { C.; Shrader, } \\
\text { Rodney C.; } \\
\text { Tompson, } \\
\text { George H. }\end{array}$ & 2006 & $\begin{array}{l}\text { - novelty of products and services } \\
\text { - TMT size } \\
\text { - TMT heterogeneity: age } \\
\text { - TMT heterogeneity: educational level } \\
\text { - TMT heterogeneity: educational } \\
\text { specialization } \\
\text { - TMT heterogeneity: functional back- } \\
\text { ground }\end{array}$ & $\begin{array}{l}\text { - } \Delta \text { sales growth vs. avg. } \\
\text { industry sales growth } \\
\text { - } \Delta \text { profitability vs. avg. } \\
\text { industry profitability } \\
\text { (mean of ROS, ROA } \\
\text { and ROE) } \\
\text { - market performance } \\
\text { (growth in price per } \\
\text { share) }\end{array}$ & $\begin{array}{l}\text { - venture age } \\
\text { - number of employees (log) } \\
\text { cal change }\end{array}$ & $\begin{array}{l}\text { - TMT size x low novelty of products and services } \\
\text { - TMT size x high novelty of products and services } \\
\text { - TMT heterogeneity: age x low novelty of products } \\
\text { and services } \\
\text { - TMT heterogeneity: age x high novelty of products } \\
\text { and services } \\
\text { - TMT heterogeneity: educational specialization x low } \\
\text { novelty of products and services } \\
\text { - TMT heterogeneity: educational specialization x high } \\
\text { novelty of products and services } \\
\text { - TMT heterogeneity: educational level x low novelty } \\
\text { of products and services } \\
\text { - TMT heterogeneity: educational level x high novelty } \\
\text { of products and services } \\
\text { - TMT heterogeneity: functional background x low } \\
\text { novelty of products and services } \\
\text { - TMT heterogeneity: functional background x high } \\
\text { novelty of products and services }\end{array}$ \\
\hline $\begin{array}{l}\text { The moderating } \\
\text { effect of environ- } \\
\text { mental dynamism } \\
\text { on the relationship } \\
\text { between entrepre- } \\
\text { neurial leadership } \\
\text { behavior and new } \\
\text { venture perform- } \\
\text { ance }\end{array}$ & $\begin{array}{l}\text { Ensley, Michael } \\
\text { D.; Pearce, Craig } \\
\text { L.; Hmieleski, } \\
\text { Keith M. }\end{array}$ & 2006 & $\begin{array}{l}\text { - environmental dynamism } \\
\text { - leadership: transactional } \\
\text { - leadership: transformational }\end{array}$ & $\begin{array}{l}\text { - sales growth } \\
\text { - sales volume }\end{array}$ & $\begin{array}{l}\text { - venture age } \\
\text { - venture size (number of } \\
\text { employees) } \\
\text { - TMT size }\end{array}$ & $\begin{array}{l}\text { - environmental dynamism x leadership: transactional } \\
\text { - environmental dynamism x leadership: transforma- } \\
\text { tional }\end{array}$ \\
\hline
\end{tabular}




\section{3}

BuR - Business Research

Official Open Access Journal of VHB

ss Research (VHB)

Volume 5 | Issue 1 | May 2012 | 83-123

Table 1 continued: Overview of the sample papers

\begin{tabular}{|c|c|c|c|c|c|c|}
\hline Title & Author(s) & Year & Independent variables & Dependent variables & Control variables & Interaction variables \\
\hline $\begin{array}{l}\text { Founders' human } \\
\text { capital and the } \\
\text { growth of new } \\
\text { technology-based } \\
\text { firms: A compe- } \\
\text { tence-based view }\end{array}$ & $\begin{array}{l}\text { Colombo, } \\
\text { Massimo G.; } \\
\text { Grilli, Luca }\end{array}$ & 2005 & $\begin{array}{l}\text { - FT avg. years of education } \\
\text { - FT avg. years of managerial education } \\
\text { - FT avg. years of technical education } \\
\text { - FT avg. years of work experience } \\
\text { - FT avg. years of work experience in } \\
\text { venture's industry } \\
\text { - FT avg. years of technical work ex- } \\
\text { perience in venture's industry } \\
\text { - FT avg. years of commercial work } \\
\text { experience in venture's industry } \\
\text { - FT avg. years of work experience in } \\
\text { other industries } \\
\text { - TMT management and leadership } \\
\text { experience } \\
\text { - TMT founding experience }\end{array}$ & - employee growth (log) & $\begin{array}{l}\text { - venture age } \\
\text { - } \text { TMT/FT size } \\
\text { - regional infrastructure de- } \\
\text { velopment index } \\
\text { - PE/VC financing received } \\
\text { - TMT motivation mainly for } \\
\text { innovative technology } \\
\text { - propensity of PE to invest in } \\
\text { venture's industry } \\
\text { - propensity of PE to invest in } \\
\text { - venture's geographic area } \\
\text { - venture industry }\end{array}$ & $\begin{array}{l}\text { - FT avg. years of technical education x FT avg. years } \\
\text { of managerial education } \\
\text { - FT avg. years of technical work experience in ven- } \\
\text { ture's industry x FT avg. years of commercial work } \\
\text { experience in venture's industry } \\
\text { - FT avg. years of technical education x FT avg. years } \\
\text { of technical work experience in venture's industry } \\
\text { - FT avg. years of technical education x FT avg. years } \\
\text { of commercial work experience in venture's industry } \\
\text { - FT avg. years of managerial education x FT avg. years } \\
\text { of technical work experience in venture's industry } \\
\text { - FT avg. years of managerial education x FT avg. years } \\
\text { of commercial work experience in venture's industry } \\
\text { - TMT management and leadership experience x FT } \\
\text { avg. years of technical education } \\
\text { - TMT management and leadership experience x FT } \\
\text { avg. years of managerial education } \\
\text { - TMT management and leadership experience x FT } \\
\text { avg. years of technical work experience in venture's } \\
\text { industry } \\
\text { - TMT management and leadership experience x FT } \\
\text { avg. years of commercial work experience in ven- } \\
\text { ture's industry }\end{array}$ \\
\hline $\begin{array}{l}\text { Management ac- } \\
\text { counting systems } \\
\text { adoption decisions: } \\
\text { Evidence and per- } \\
\text { formance implica- } \\
\text { tions from early- } \\
\text { stage/startup com- } \\
\text { panies }\end{array}$ & $\begin{array}{l}\text { Davila, Antonio; } \\
\text { Foster, George }\end{array}$ & 2005 & $\begin{array}{l}\text { - Industry biotechnology } \\
\text { - Industry information technology } \\
\text { - Funding received } \\
\text { - FT member replaced } \\
\text { - CEO planning beliefs } \\
\text { - Adoption decisions }\end{array}$ & $\begin{array}{l}\text { - Employee growth } \\
\text { - Sales growth }\end{array}$ & & - \\
\hline
\end{tabular}




\section{3}

BuR - Business Research

Official Open Access Journal of VHB

ess Research (VHB)

Volume 5 | Issue 1 | May 2012 | 83-123

Table 1 continued: Overview of the sample papers

\begin{tabular}{|c|c|c|c|c|c|c|}
\hline Title & Author(s) & Year & Independent variables & Dependent variables & Control variables & Interaction variables \\
\hline $\begin{array}{l}\text { A comparative study } \\
\text { of new venture top } \\
\text { management team } \\
\text { composition, dy- } \\
\text { namics and per- } \\
\text { formance between } \\
\text { university-based } \\
\text { and independent } \\
\text { start-ups }\end{array}$ & $\begin{array}{l}\text { Ensley, Michael } \\
\text { D.; Hmieleski, } \\
\text { Keith M. }\end{array}$ & 2005 & $\begin{array}{l}\text { - TMT potency } \\
\text { - TMT cohesion } \\
\text { - TMT shared strategic cognition and } \\
\text { consensus } \\
\text { - TMT idea conflict } \\
\text { - TMT relationship conflict } \\
\text { - TMT heterogeneity }\end{array}$ & $\begin{array}{l}\text { - sales growth }(\log ) \\
\text { - net cash flows }(\log )\end{array}$ & $\begin{array}{l}\text { - venture age } \\
\text { - number of employees (log) } \\
\text { - TMT size }\end{array}$ & - \\
\hline $\begin{array}{l}\text { Demographic diver- } \\
\text { sity for building an } \\
\text { effective entrepre- } \\
\text { neurial team: is it } \\
\text { important? }\end{array}$ & $\begin{array}{l}\text { Chowdhury, } \\
\text { Sanjib }\end{array}$ & 2005 & $\begin{array}{l}\text { - TMT heterogeneity: age } \\
\text { - TMT heterogeneity: gender } \\
\text { - TMT heterogeneity: functional back- } \\
\text { ground } \\
\text { - TMT cohesion: belonging and com- } \\
\text { mitment } \\
\text { - TMT cognitive comprehensiveness }\end{array}$ & - team effectiveness & - TMT size & - \\
\hline $\begin{array}{l}\text { Antecedents, mod- } \\
\text { erators, and per- } \\
\text { formance conse- } \\
\text { quences of member- } \\
\text { ship change in new } \\
\text { venture teams }\end{array}$ & $\begin{array}{l}\text { Chandler, Gay- } \\
\text { len N.; Honig, } \\
\text { Benson; } \\
\text { Wiklund, Johan }\end{array}$ & 2005 & $\begin{array}{l}\text { - TMT size } \\
\text { - environmental dynamism } \\
\text { - venture stage of development } \\
\text { - TMT heterogeneity: educational } \\
\text { specialization } \\
\text { - TMT heterogeneity: years of industry } \\
\text { experience } \\
\text { - TMT heterogeneity: gender } \\
\text { - TMT heterogeneity: religious affilia- } \\
\text { tion } \\
\text { - TMT heterogeneity: political party } \\
\text { - TMT heterogeneity: functional back- } \\
\text { ground } \\
\text { - TMT additions } \\
\text { - TMT departures }\end{array}$ & $\begin{array}{l}\text { - sales growth } \\
\text { - profitability } \\
\text { - turnover: additions } \\
\text { - turnover: departures }\end{array}$ & $\begin{array}{l}\text { - industry: service } \\
\text { - industry: trading } \\
\text { - industry: manufacturing } \\
\text { - industry: retail } \\
\text { - industry: business services } \\
\text { - venture age }\end{array}$ & $\begin{array}{l}\text { - } \quad \text { venture stage of development x turnover: additions } \\
\text { - } \quad \text { enture stage of development x turnover: departures } \\
\text { - } \quad \text { environmental dynamism x turnover: additions } \\
\text { xiral dynamism x turnover: departures }\end{array}$ \\
\hline
\end{tabular}




\section{3}

BuR - Business Research

Official Open Access Journal of VHB

ess Research (VHB)

Volume 5 | Issue 1 | May 2012 | 83-123

Table 1 continued: Overview of the sample papers

\begin{tabular}{|c|c|c|c|c|c|c|}
\hline Title & Author(s) & Year & Independent variables & Dependent variables & Control variables & Interaction variables \\
\hline $\begin{array}{l}\text { Signaling in Venture } \\
\text { Capitalist - New } \\
\text { Venture Team } \\
\text { Funding Decisions: } \\
\text { Does It Indicate } \\
\text { Long-Term Venture } \\
\text { Outcomes? }\end{array}$ & $\begin{array}{l}\text { Busenitz, Lowell } \\
\text { W.; Fiet, James } \\
\text { O.; Moesel, } \\
\text { Douglas D. }\end{array}$ & 2005 & $\begin{array}{l}\text { - } \text { equity held by TMT } \\
\text { - TMT wealth invested }\end{array}$ & $\begin{array}{l}\text { - venture's exit status } \\
\text { (out of business, still } \\
\text { private, merged or ac- } \\
\text { quired, IPO) }\end{array}$ & $\begin{array}{l}\text { - } \text { year of VC funding } \\
\text { - } \text { volume of VC funding } \\
\text { - venture age } \\
\text { - } \text { sales } \\
\text { - TMT firm-specific skills } \\
\text { - TMT founding experience } \\
\text { - TMT VC experience } \\
\text { - TMT industry experience }\end{array}$ & - \\
\hline $\begin{array}{l}\text { Experience-based } \\
\text { top management } \\
\text { team competence } \\
\text { and sustained } \\
\text { growth }\end{array}$ & Kor, Yasemin Y. & 2003 & $\begin{array}{l}\text { - founders in TMT } \\
\text { - TMT acquaintance } \\
\text { - TMT industry experience }\end{array}$ & - sales growth & $\begin{array}{l}\text { - TMT avg. age } \\
\text { - TMT size } \\
\text { - TMT tenure heterogeneity } \\
\text { - venture age } \\
\text { - } \text { total assets } \\
\text { - years since IPO }\end{array}$ & $\begin{array}{l}\text { - founders in TMT x TMT acquaintance } \\
\text { - founders in TMT x TMT industry experience }\end{array}$ \\
\hline $\begin{array}{l}\text { Organizational } \\
\text { endowments and } \\
\text { the performance of } \\
\text { university start-ups }\end{array}$ & $\begin{array}{l}\text { Shane, Scott; } \\
\text { Stuart, Toby }\end{array}$ & 2002 & $\begin{array}{l}\text { - } \text { patent stock at founding } \\
\text { - patent effectiveness } \\
\text { - right to use exclusive technology } \\
\text { license } \\
\text { - prestige of underlying technology } \\
\text { - TMT industry experience } \\
\text { - TMT founding experience } \\
\text { - industry size at founding } \\
\text { - TMT direct tie to investor } \\
\text { - TMT indirect tie to investor }\end{array}$ & $\begin{array}{l}\text { - IPO } \\
\text { - Venture exit } \\
\text { - time to receive VC } \\
\text { funding }\end{array}$ & $\begin{array}{l}\text { - IPO rate in industry } \\
\text { - IPO count in industry } \\
\text { - Semiconductor industry } \\
\text { - Massachusetts Institute of } \\
\text { Technology invested in ven- } \\
\text { ture } \\
\text { - Cumulative VC funds raised } \\
\text { by venture } \\
\text { - Sum of invested money by } \\
\text { Small Business Research In- } \\
\text { vestment funds } \\
\text { - Cumulative revenues by } \\
\text { venture (log) }\end{array}$ & - \\
\hline $\begin{array}{l}\text { Understanding the } \\
\text { dynamics of new } \\
\text { venture top man- } \\
\text { agement teams } \\
\text { cohesion, conflict, } \\
\text { and new venture } \\
\text { performance }\end{array}$ & $\begin{array}{l}\text { Ensley, Michael } \\
\text { D.; Pearson, } \\
\text { Allison W.; } \\
\text { Amason, Allen } \\
\text { C. }\end{array}$ & 2002 & $\begin{array}{l}\text { - TMT cognitive conflict } \\
\text { - TMT affective conflict } \\
\text { - TMT cohesion: belonging and com- } \\
\text { mitment } \\
\text { - TMT cohesion: morale }\end{array}$ & $\begin{array}{l}\text { - sales growth } \\
\text { - profitability }\end{array}$ & $\begin{array}{l}\text { - venture age } \\
\text { - number of employees } \\
\text { - TMT size }\end{array}$ & - \\
\hline
\end{tabular}




\section{3}

BuR - Business Research

Official Open Access Journal of VHB

ess Research (VHB)

Volume 5 | Issue 1 | May 2012 | 83-123

Table 1 continued: Overview of the sample papers

\begin{tabular}{|c|c|c|c|c|c|c|}
\hline Title & Author(s) & Year & Independent variables & Dependent variables & Control variables & Interaction variables \\
\hline $\begin{array}{l}\text { Shared cognition in } \\
\text { top management } \\
\text { teams: implications } \\
\text { for new venture } \\
\text { performance }\end{array}$ & $\begin{array}{l}\text { Ensley, Michael } \\
\text { D.; Pearce, Craig } \\
\text { L. }\end{array}$ & 2001 & $\begin{array}{l}\text { - TMT cognitive conflict } \\
\text { - TMT affective conflict } \\
\text { - TMT shared strategic cognition and } \\
\text { consensus } \\
\text { - TMT cohesion: belonging and com- } \\
\text { mitment } \\
\text { - TMT cohesion: morale }\end{array}$ & $\begin{array}{l}\text { - sales growth } \\
\text { - profitability } \\
\text { - sales volume }\end{array}$ & - & - \\
\hline $\begin{array}{l}\text { The effect of entre- } \\
\text { preneurial team skill } \\
\text { heterogeneity and } \\
\text { functional diversity } \\
\text { on new venture } \\
\text { performance }\end{array}$ & $\begin{array}{l}\text { Ensley, Michael } \\
\text { D.; Carland, } \\
\text { James W.; } \\
\text { Carland, JoAnn } \\
\text { C. }\end{array}$ & 1998 & $\begin{array}{l}\text { - TMT heterogeneity: functional back- } \\
\text { ground } \\
\text { - TMT heterogeneity: educational level } \\
\text { - TMT heterogeneity: educational } \\
\text { specialization }\end{array}$ & $\begin{array}{l}\text { - sales volume } \\
\text { - sales growth } \\
\text { - profitability }\end{array}$ & - TMT size & - \\
\hline $\begin{array}{l}\text { Cooperative strategy } \\
\text { and new venture } \\
\text { performance: the } \\
\text { role of business } \\
\text { strategy and man- } \\
\text { agement experience }\end{array}$ & $\begin{array}{l}\text { McGee, Jeffrey } \\
\text { E.; Dowling, } \\
\text { Michael J.; } \\
\text { Megginson, } \\
\text { William L. }\end{array}$ & 1995 & $\begin{array}{l}\text { - TMT marketing experience (abs. and } \\
\text { rel.) } \\
\text { - TMT technical experience (abs. and } \\
\text { rel.) } \\
\text { - TMT production experience (abs. and } \\
\text { rel.) } \\
\text { - marketing cooperation } \\
\text { - technical cooperation } \\
\text { - production cooperation } \\
\text { - } \text { pursued venture strategy }\end{array}$ & - sales growth (log) & $\begin{array}{l}\text { - venture age } \\
\text { - total assets }\end{array}$ & $\begin{array}{ll}\text { - } & \text { marketing cooperation x TMT marketing experience } \\
\text { (abs.) } & \\
\text { - } & \text { (rel.) } \\
\text { - } & \text { technical cooperation x TMT technical experience } \\
& \text { (abs.) } \\
\text { - } & \text { technical cooperation x TMT technical experience } \\
\text { (rel.) } \\
\text { - } \\
\text { production cooperation x TMT production experi- } \\
\text { ence (abs.) } \\
\text { - production cooperation x TMT production experi- } \\
\text { ence (rel.) }\end{array}$ \\
\hline $\begin{array}{l}\text { Using R\&D coop- } \\
\text { erative arrange- } \\
\text { ments to leverage } \\
\text { managerial experi- } \\
\text { ence: a study of } \\
\text { technology- } \\
\text { intensive new ven- } \\
\text { tures }\end{array}$ & $\begin{array}{l}\text { McGee, Jeffrey } \\
\text { E.; Dowling, } \\
\text { Michael J. }\end{array}$ & 1994 & $\begin{array}{l}\text { - TMT industry experience } \\
\text { - TMT technical experience } \\
\text { - technical cooperation }\end{array}$ & - sales growth & $\begin{array}{l}\text { - venture age } \\
\text { - venture size (total assets) }\end{array}$ & $\begin{array}{l}\text { - TMT industry experience } \mathrm{x} \text { technical cooperation } \\
\text { - TMT technical experience } \mathrm{x} \text { technical cooperation }\end{array}$ \\
\hline
\end{tabular}




\section{3}

BuR - Business Research

Official Open Access Journal of VHB

ess Research (VHB)

Volume 5 | Issue 1 | May 2012 | 83-123

Table 1 continued: Overview of the sample papers

\begin{tabular}{|c|c|c|c|c|c|c|}
\hline Title & Author(s) & Year & Independent variables & Dependent variables & Control variables & Interaction variables \\
\hline $\begin{array}{l}\text { Initial human and } \\
\text { financial capital as } \\
\text { predictors of new } \\
\text { venture perform- } \\
\text { ance }\end{array}$ & $\begin{array}{l}\text { Cooper, Arnold } \\
\text { C.; Gimeno- } \\
\text { Gascon, F. } \\
\text { Javier; Woo, } \\
\text { Carolyn Y. }\end{array}$ & 1994 & $\begin{array}{l}\text { - TMT level of education } \\
\text { - TMT gender } \\
\text { - TMT members consist of minority group } \\
\text { - parents of TMT members owned small } \\
\text { business } \\
\text { - TMT members employed at non-profit } \\
\text { - } \text { TMT before founding } \\
\text { - use of professiont experience } \\
\text { - TMT size } \\
\text { - TMT industry experience } \\
\text { - initial capital at founding }\end{array}$ & $\begin{array}{l}\text { - employee growth } \\
\text { - venture survival }\end{array}$ & - industry sector & - \\
\hline $\begin{array}{l}\text { Organizational } \\
\text { growth: linking } \\
\text { founding team, } \\
\text { strategy, environ- } \\
\text { ment, and growth } \\
\text { among U.S. semi- } \\
\text { conductor ventures, } \\
\text { 1978-1988 }\end{array}$ & $\begin{array}{l}\text { Eisenhardt, } \\
\text { Kathleen M.; } \\
\text { Schoonhoven, } \\
\text { Claudia Bird }\end{array}$ & 1990 & $\begin{array}{l}\text { - TMT size } \\
\text { - TMT acquaintance } \\
\text { - TMT heterogeneity: years of industry } \\
\text { experience } \\
\text { - growth market } \\
\text { - competitive concentration } \\
\text { - technical innovation } \\
\text { - TMT strength (combination of size, ac- } \\
\text { quaintance, and heterogeneity) }\end{array}$ & - sales growth & $\begin{array}{l}\text { - venture age } \\
\text { - market size } \\
\text { - initial capital at founding } \\
\text { - book to bill ratio (semi- } \\
\text { conductor industry) }\end{array}$ & $\begin{array}{l}\text { - venture age x TMT heterogeneity: years of industry } \\
\text { - experience } \\
\text { - venture age x TMT size } \\
\text { - venture age x TMT acquaintance } \\
\text { - TMT strength x growth market } \\
\text { - venture age x growth market } \\
\text { - venture age x TMT strength } \\
\text { - venture age x TMT strength } x \text { growth market }\end{array}$ \\
\hline $\begin{array}{l}\text { Impact of entrepre- } \\
\text { neurial and man- } \\
\text { agement experience } \\
\text { on early perform- } \\
\text { ance }\end{array}$ & $\begin{array}{l}\text { Stuart, Robert } \\
\text { W.; Abetti, Pier } \\
\text { A. }\end{array}$ & 1990 & $\begin{array}{l}\text { - strategy } \\
\text { - } \text { organization } \\
\text { - stage in product life cycle } \\
\text { - TMT entrepreneur type } \\
\text { - TMT founding experience } \\
\text { - TMT prior management level } \\
\text { - TMT leadership experience } \\
\text { - TMT avg. age } \\
\text { - TMT management experience } \\
\text { - TMT technical experience } \\
\text { - TMT marketing experience } \\
\text { - TMT financial experience } \\
\text { - TMT education } \\
\text { - TMT total experience }\end{array}$ & $\begin{array}{l}\text { - performance (measure } \\
\text { of sales growth, per- } \\
\text { formance growth, prof- } \\
\text { itability, and productiv- } \\
\text { ity) }\end{array}$ & - & - \\
\hline
\end{tabular}


resources and social ties, facilitates environmental scanning, and offers more information sources to draw from (Amason, Shrader, and Tompson 2006). This is of special importance, as start-ups have less room for duplication in the management's skill set (Hmieleski and Ensley 2007). Investors and venture capitalists not only consider financial figures and markets, but also the quality and composition of the team in their evaluation process. In particular, broad functional experience which goes along with functional diversity attracts investors and external stakeholders (Beckman, Burton, and O’Reilly 2007). On the other hand, heterogeneity can induce increased interpersonal conflict and emotions and disrupt group processes resulting from diverging perspectives and experience (Beckman, Burton, and O'Reilly 2007; Amason and Sapienza 1997). While affective conflict has a negative influence on group cohesion (Chowdhury 2005), cognitive conflict is seen as a facilitator for a comprehensive shared view on the firm (Ensley and Pearce 2001). Summarizing these theoretical aspects, it can be said that an interpretation of team heterogeneity always has to pay attention to the given context it depends on, the purpose and strategy of the firm, as well as the environmental conditions (e.g., market dynamics, industry) (Hmieleski and Ensley 2007).

Heterogeneity in general was tested by Ensley and Hmieleski (2005) and Hmieleski and Ensley (2007) via an index of educational level, educational specialization, functional expertise, and general managerial skills. Ensley and Hmieleski (2005) found a positive impact of general team heterogeneity on sales growth of a national sample of new hightechnology ventures. Hmieleski and Ensley (2007) instead revealed a negative relationship between team heterogeneity and an index of sales and employee growth for both a national random sample and an Inc. 500 (500 fastest-growing private companies) sample. As the measurement of heterogeneity and the sample of the national random sample appear to be the same in both studies, the difference in impact direction might be attributed to the dependent variable. As a higher degree of heterogeneity supplies the firm with a broader range of covered skills and capabilities, the necessity to grow in terms of employees is less necessary and thereby reduces the impact on a compound growth index (including employee growth) as applied by Hmieleski and Ensley (2007). However, the analysis of interaction variables draws a more differentiated picture. The negative effect of heterogeneity is even worse when empowering leadership is applied in high-dynamic environments, while the negative effect declines and is almost neutral when empowering leadership is applied in low-dynamic environments. As for directive leadership this effect turns around.

Age heterogeneity was investigated by Amason, Shrader, and Tompson (2006), arguing that team members of similar age are more likely to share common experience (Wiersema and Bantel 1992). While age heterogeneity and high venture novelty interact negatively on sales growth, the combination with low novelty shows a positive relationship.

Amason, Shrader, and Tompson (2006) and Ensley, Carland, and Carland (1998) tested on educationallevel heterogeneity, revealing contradictory results on sales growth. A positive influence was found by Amason, Shrader, and Tompson (2006), although this effect is neutralized when educational level heterogeneity interacts with high venture novelty. In contrast, results of Ensley, Carland, and Carland (1998) showed the opposite effect. Both studies differ in the heterogeneity measurement method and the underlying sample. While Amason, Shrader, and Tompson's (2006) study was based on firms that issued initial public offerings, Ensley, Carland, and Carland (1998) used Inc. 500 ventures. Future research endeavors should thereby control at least for IPOs and above-average performance among the sample and should apply different heterogeneity measures. The effect on capital raised via IPOs was found by Zimmerman (2008) to be positively influenced by functional heterogeneity.

Vissa and Chacar (2009) revealed a positive relationship between functional background heterogeneity and sales growth. Instead, Amason, Shrader, and Tompson (2006) found detrimental effects in case of combination with high venture novelty. A further distinction between FTs and TMTs was made by Beckman, Burton, and O’Reilly (2007). While there is a positive influence of functional background heterogeneity on venture capital funding for both, the influence on the rate of going public is weakly negative for FTs only. Zimmerman (2008) instead found positive effects on the capital raised via IPO for TMTs. Thus, functional background heterogeneity revealed a set of contradictory findings where further detailing is recommended. Moreover, Eisenhardt and Schoonhoven (1990) revealed a positive impact of industry experience heterogeneity on sales growth. The positive effect 
grows with the age of the firm. However, further studies are suggested to control for dynamic influencing factors such as environmental dynamism or venture novelty.

\subsection{Influence of Team Leadership}

In established organizations the structures, rules, and work-processes function as an orientation for employees and are already well defined. In entrepreneurial environments such an orientation is not yet established and mainly provided by founders and top managers, enforced by their individual leadership style. Previous studies distinguished leadership dimensions in empowering vs. directive or transformational vs. transactional leadership (Pearce, Sims, Cox, Ball, Schnell, Smith, and Trevino 2003).

Empowering leadership motivates, encourages, and supports followers to release individual strengths, intrinsic motivations, and confidence. As a result, independent behavior, participative goal-setting, opportunity thinking, and self-leadership lead to team potency and self-efficacy (Spreitzer 1996). In an entrepreneurial context, such outcomes of leadership can help to maintain the necessary motivation and commitment to compete against more established and resource-rich firms (Ensley, Pearson, and Pearce 2003). On the other hand, uncoordinated activities not leading to the desired results and incompatibility of initiatives and tasks can generate inefficiencies and counterproductive outcomes (Gebert, Boerner, and Lanwehr 2003; Hmieleski and Ensley 2007). These implications can become especially critical in heterogeneous teams and dynamic environments as defined goals, processes and a collective vision are necessary to coordinate diverse team members efficiently and fast. Directive leadership in contrast is a more appropriate approach to fulfill these requirements. However, recent studies focused primarily on negative impacts, as instructions, commands, and assignment of nonnegotiable goals and tasks reveal some disruptive effects on a team's performance (Pearce, Sims, Cox, Ball, Schnell, Smith, and Trevino 2003). Because of directive leadership creativity and motivations can be repressed, diverse perspectives cannot incubate and develop, different views are not taken into consideration, and conflicts can arise from top-down orders (Hmieleski and Ensley 2007).

Transformational approaches focus on a vision to follow and encourage followers toward self-efficacy and idealism. Instead, transactional leaders implement contingent material and personal rewards and lead by rational stimulation (e.g., Pearce, Sims, Cox, Ball, Schnell, Smith, and Trevino 2003; Rafferty and Griffin 2004).

As some leadership effects in connection with a team's heterogeneity were already presented above, this section covers leadership types alone and interactions with environmental dynamism. Hmieleski and Ensley (2007) and Ensley, Pearce, and Hmieleski (2006) discovered significant relationships on a venture growth index and sales growth respectively. Positive links were found for directive and transactional and negative links for empowering and transformational leadership. As expected, in combination with high environmental dynamics, empowering and transformational leadership styles seem to outperform directive and transactional styles in terms of growth. Moreover, Ensley, Hmieleski, and Pearce (2006) distinguished between vertical, which is topdown leadership by an individual, and shared leadership, which is collaborative leadership in a team. With shared leadership the negative influence of transformational and empowering leadership turns positive, while the positive influence of directive and transactional leadership is confirmed for vertical and shared leadership.

\subsection{Influence of Team Turnover}

A firm's TMT is not an everlasting stable construct; instead it is subject to team turnover, which implicates tenure heterogeneity (Ucbasaran, Lockett, Wright, and Westhead 2003). The composition and the number of members frequently change over time and adapt to new corporate challenges, strategic adjustments, and a changing environment (Boeker 1997). Reasons can be new requirements in the management's skills, new strategic orientation, founders switching to the supervisory board, low performance of individual members, pressure from capital investors, or political reasons.

Effort for replacement (e.g., recruiting, introduction phases, hiring, low initial productivity) of departed managers can become a costly affair (Dess and Shaw 2001). Furthermore, tacit and explicit knowledge are valuable resources of the firm and no longer contribute to and are utilized in the interest of the firm. In an environment where efforts are not valued and targets are not achieved, poor performers are more likely to leave a firm than good performers (Chandler, Honig, and Wiklund 2005; 
McEvoy and Cascio 1987). Some scholars interpreted departing managers as a possible signal of internal problems (Beckman, Burton, and O'Reilly 2007). High-quality managers might depart in order to obtain better opportunities or get rid of an economically or socially unsatisfying situation.

Adding team members can also be accompanied with negative effects on a team's cohesion and integrity. New team members can lead to disruption, as young entrepreneurial groups operate more informally with tacit cohesion and existing experience of how to work together in a team (Chandler, Honig, and Wiklund 2005). Taking the time for functional integration and adaptation, considerable socialization, and a clear collective understanding of roles, tasks, and responsibilities are of major importance (Kemery, Bedeian, Mossholder, and Touliatos 1985; Heinen and Jacobson 1976). In contrast, added members tend to enrich a firm with new information sources, skills, network ties, or resources available, and therefore influence a firm's performance. As teams with experience in working together operate more effectively and efficiently, such teams might have an advantage under stable conditions. In contrast, more dynamic and turbulent conditions give a firm the opportunity to learn from new team members and adapt to changes and therefore be advantageous (Murray 1989).

Chandler, Honig, and Wiklund (2005) distinguished between added and departing team members and tested for impacts on sales growth. Positive influence on sales growth was found by the interaction of a high stage of a firm's development and departing team members, as well as by high industry dynamism and the addition of new team members. The opposite effect appears in case of the interaction of a high stage of a firm's development and the addition of new members, as well as high industry dynamics and departing team members. High dynamics call for an increase of human and social capital for being able to cope with environmental challenges. Due to the pervasive lifecycle of a firm, a founder's skill set can become inadequate to rising corporate challenges a firm must face as it grows and matures. Thus, new team members with superior matching skills need to replace existing team members (Boeker and Wiltbank 2005; Rubenson and Gupta 1996). Therefore, Beckman, Burton, and O’Reilly (2007) and Beckman and Burton (2008) differentiated between total TMT entrants, total TMT exits, and founder exits and test on going pub- lic and VC funding. Total TMT entrants and founder exits show a strong positive influence on going public, while total TMT exits have positive effects and TMT entrants negative effects on VC funding. Once the founder replacements have been accounted for, departures from the team have a negative effect on the rate of going public. Instead, Davila and Foster (2005) found the replacement of founders to have negative influence on employee growth. This might be reasoned by a more efficiency-oriented governance approach of a more professional replacement management.

\subsection{Influence of Team Dynamics}

An ETMT's dynamics are distinguished in a conflict component and a cohesion component. Conflict can be separated into cognitive and affective conflict (e.g., Ensley, Pearson, and Amason 2002) or idea and relationship conflict (e.g., Jehn 1995). Cohesion is determined by indicators such as sense of belonging and commitment, morale, shared strategic cognition, and consensus, as well as a team's potency. Due to the novelty and not yet established rules and organizational structures of new ventures, several perspectives, conflicting ideas, and divergent perceptions arise. Ensley and Pearce (2001) described conflict as a process that teams go through to make decisions, take action, and create cognitive schemata. Especially in entrepreneurial teams conflict is common, as tasks are challenging, innovative, and involve high risks for individuals and the team (Gartner 1990; Hornaday 1992). Ensley and Pearce (2001) and Ensley and Hmieleski (2005) described cognitive conflict as a group process of thinking about multiple ideas, sharing these ideas, and developing them through constructive discussion and argument with a certain level of disagreement. Cognitive conflict facilitates the exchange of ideas, the contrasting of divergent perspectives, and the objective assessment of alternatives. Thereby it delivers creative ideas and solutions (Ensley, Pearson, and Amason 2002), as long as an open and respectful culture of interaction is maintained (Ensley and Hmieleski 2005).

When cognitive conflicts are misinterpreted or inflamed due to divergent values, suspicion, different perspectives, or mistrust, affective conflict can be a result even in case of moderate and reasonable criticism (Baron 1988; Pelled 1996). This can be caused by frequent negative cognitive conflict which leads to high emotional displeasure and thus can result in 
irrational disagreement and increasing infighting (Ensley and Hmieleski 2005). Affective conflict is emotional, carried out on a personal level, can lead to anger and alienation, and extends beyond different opinions of team members and beyond the team's purpose (Ensley and Hmieleski 2005). Summarizing, teams are advised to embrace the benefits of cognitive conflict and avoid the costs and frictions of affective conflict in order to increase effectiveness.

As expected, Ensley and Pearce (2001), Ensley, Pearson, and Amason (2002), and Ensley and Hmieleski (2005) found negative relationships between affective conflict and sales growth. Furthermore, a positive influence of cognitive conflict on sales growth was found by Ensley and Pearce (2001) and Ensley and Hmieleski (2005), whereas Ensley, Pearson, and Amason (2002) could not confirm this link. These results have to be interpreted against the fact of similar samples among Ensley and Pearce (2001) and Ensley, Pearson, and Amason (2002) and similar measurement methods of affective and cognitive conflict across all three studies. Specific sample characteristics and potential common method bias forbids interpreting these findings of seemingly strong collective conformity at first sight as three independent tests.

A team's cohesion is seen as a strong facilitator of cognitive conflict in decision-making processes, as members are expected to be more open to dissent, discussions, and criticism (Ensley and Pearce 2001). The team members exhibit higher levels of affinity, affective attraction, and trust amongst each other and are more satisfied with the group's setup as a whole (O'Reilly III, Caldwell, and Barnett 1989). Team cohesion is the perceived affective sense of morale, belonging (Bollen and Hoyle 1990), commitment, consensus, and a strong belief in the team's potency among members. Such teams share a tacit understanding and values, do not need to spend energy on group maintenance and frictions, and gain from synergies (Smith, Smith, Sims, O’Bannon, Scully, and Olian 1994). Developing a shared understanding and consensus is the result of discussions of strategic alternatives and the associated cognitive conflict (e.g., Amason 1996). Cohesion facilitates speedy collective action, group interaction, resource sharing among team members, and improves communication and team efficiency (Vissa and Chacar 2009). This is crucial for entrepreneurial teams, due to the ambiguity and complexity of their tasks (Ensley and Pearce 2001; West 2007). On the other hand, strong personal attraction might lead to an uncritical and complacent view of the other team member's work, performance, and conception, thus leading to failure or suboptimal decisions.

Positive relationships on sales growth were found by Ensley and Hmieleski (2005) when testing for the influence of general team cohesion, shared strategic cognition, and team potency, all with significant influence. Team cohesion variables such as belonging and commitment, as well as morale, were tested by Ensley and Pearce (2001), Ensley, Pearson, and Amason (2002), and Chowdhury (2005). While Ensley and Pearce (2001) were able to reveal indirect effects via conflict variables only, Ensley, Pearson, and Amason (2002) found a clear impact of belonging and commitment on sales growth. This is in line with Chowdhury (2005), who discovered a significant influence of belonging and commitment on team effectiveness, which is expected to influence sales growth positively. There is strong collective agreement on the positive impact of ETMT cohesion on new venture sales growth among the reviewed sample. As the measurement method of cohesion variables is equal for Ensley and Hmieleski (2005) and Ensley, Pearson, and Amason (2002), the reader should be aware of potential (but not expected by the authors) common method bias.

Vissa and Chacar (2009), in addition, investigated the interaction effects of cohesion and a team's network constraints on sales growth. The latter describes the inability to span structural holes of a team's network and therefore reduces the likelihood to access a broad array of resources. The combination of strong cohesion and network constraints amplify the delimitation from external sources and thus influence growth negatively.

\subsection{Influence of Team Experience}

ETMT's experience in industries, functions, work groups, or founding endeavors is an important determinant of a team's competencies. A manager's experience can mirror the skills, knowledge, resources, and social ties available to the team (Harris and Helfat 1997; Castanias and Helfat 2001). Shrader and Siegel (2007) saw increased worker productivity, higher economic value of the firm, and growth in managerial compensation as a result of experience. The knowledge, networks, and judgment skills of experienced managers lead to a supe- 
rior availability of information and allows for making more comprehensive strategic decisions and gaining competitive advantages. Such human capital can be proxied by years of work, prior companies, and industry or managerial experience (Colombo and Grilli 2005). Another suitable proxy is the average age of team members which is explained in further detail in the chapter 4.1Influence of Team Demographics. Apart from the positive influences of experience among the ETMT, the combination of diverse experiences can lead to conflicting configurations (Meyer, Tsui, and Hinings 1993), which are additionally investigated in some of the sample papers (e.g., Kor 2003).

Managers can leverage their work experience and bring several contacts, diverse experiences, and tacit knowledge about managing and organizing work processes from prior company affiliations that contribute to the new firm's social capital and benefit the firm. Such social capital contributes to the bridging effect (Adler and Kwon 2002), which results from external relations and links an organization to external resources and information and extends an organization's scope, coverage, and range. For example, Almus and Nerlinger (1999) revealed a negative influence of entrepreneurs without professional experience at start-up time on a firm's growth.

A positive impact of the average years of founders' work experience on employee growth was found by Colombo and Grilli (2005), independent of the industry of gathered experience. When segmenting, there are positively amplifying interacting effects between industry-specific commercial work experience and industry-specific technical work experience. Following Beckman, Burton, and O'Reilly (2007), the number of prior companies of the ETMT, which can be seen as a measure for an ETMT network's breadth, influences positively both VC funding and going public, while there were no findings for the FT. Instead, the number of overlapping prior companies (where more than one team member was employed) determines positively the chance of going public for FTs.

Managers with firm-specific industry experience can benefit a firm, as they are able to introduce competitive trends and conditions, specific technologies, industry-specific regulatory issues, or goodwill with certain customers and suppliers (Kor 2003). Furthermore, the superior identification of emerging opportunities, threats, and potential applications in the market, as well as a reasoned posi- tion of new products and services tailored to suit market needs, increase productivity and reduce transaction costs (McGee, Dowling, and Megginson 1995; Castanias and Helfat 2001). Such consciousness about an industry and social ties to it may significantly reduce the liability of newness that entrepreneurial firms may otherwise have due to their underdeveloped connections to suppliers and customers as well as due to such firms' missing establishment in the market (Stinchcombe 1965).

Shrader and Siegel (2007) surprisingly found a negative impact of experience in the industry similar to the new venture on sales growth. This might be induced by the limited scope on technology-based publicly traded new ventures where functional experience is more relevant and would suffer from high presence of industry specialists. In contrast, other authors collectively agreed on the opposite effect and showed strong positive influences. Shane and Stuart (2002) showed positive effects on going public; Colombo and Grilli (2005) and Cooper, Gimeno-Gascon, and Woo (1994) on employee growth; and McGee and Dowling (1994) on sales growth - the latter especially in combination with technical cooperation. Although there is strong evidence from the review sample, future research is suggested to focus on the capabilities which have to be sacrificed for industry experience and how this might result in detrimental effects.

Managers with more functional experience claim a resource that can contribute to a firm's ability to define and implement specific strategies (Shrader and Siegel 2007). Thus, it is suggested that functional experience of an ETMT is reflected in the type of strategy pursued by a new venture. Firms with a good fit between functional experience and the pursued competitive strategy are expected to enjoy better performance. However, management and leadership experience in general was found to relate positively to the rate of VC funding and IPO (Beckman, Burton, and O'Reilly 2007; Beckman and Burton 2008), as well as to a performance and growth index (Gimeno, Folta, Cooper, and Woo 1997).

McGee, Dowling, and Megginson (1995), McGee and Dowling (1994), and Shrader and Siegel (2007) showed that the combination with marketing cooperation or a pursued differentiation strategy significantly enhances the impact of marketing experience on the firm's sales growth. The same applies to technological experience with technological coop- 
eration and production experience with production cooperation and according strategies. However, technological cooperation with inexperienced managers lead to lower sales growth rates compared to no cooperation. As suggested by Shrader and Siegel (2007), not the functional experience itself, but the appropriate fit between functional experience and pursued strategy or cooperation is key to superior performance, enabling the utilization of available skills to a significant extent.

Founders are a source of creativity and productive commercialization of ideas, technologies, and needs (Hsu 2007). Therefore, they combine important characteristics for the successful establishment of a new venture. Managers obtain founding experience via prior self-employment, working as an investor for new ventures, or working as a manager with corporate entrepreneurial responsibilities. Following Kaplan and Strömberg (2004), founding experience is an important positive factor perceived by VCs for investment decisions due to a proven track record and thus should be advantageous for new ventures to receive funding. Prior founding success is likely to send a signal of entrepreneurial quality, business development abilities, and existing network contacts to potential investors (Hsu 2007). In addition, it provides the team with knowledge about necessary and relevant actions to be taken, potential hurdles, and critical success factors within the founding process.

Several sample papers revealed the positive influence of founding experience in the ETMT on the firm. Colombo and Grilli (2005) found a relation to employee growth; Beckman, Burton, and O'Reilly (2007) to the probability of going public for TMTs and FTs; and Hsu (2007) to the valuation of new ventures right before VC funding. Hsu (2007) also found a positive relationship between the recruiting rate of executives via the founders' personal network and the valuation of a new venture right before $\mathrm{VC}$ funding. This signals a broad network, social ties, and a greater range of available resources and therefore leads to higher expectations. Surprisingly, Shane and Stuart (2002) found evidence for negative influence of founding experience on VC funding. As the sample considers firms founded at the Massachusetts Institute of Technology only, one explanation might be that founding experience among the ETMT signals unsuccessful prior founding endeavors, as the ETMT members obviously were not able to stay as a manager in the firm and returned to university.

Shared team-specific experience in managerial activities is the tacit knowledge of each team member regarding personal skills, limitations, and typical habits gathered through interaction, debates, working together, and discussions (Eisenhardt and Schoonhoven 1990; Penrose 1959). Such teams are able to save time in coordination and are prepared for higher risk taking, due to better communication within the team and more efficient and successful decision-making. This enables the team to focus on business issues rather than on interpersonal conflicts (e.g., Kor and Mahoney 2000; Jackson 1992). The results are reduced goal conflict within the team, improved socialization, positive team dynamics, increased communication, group cohesion, less friction, and lower team turnover (e.g., Smith, Smith, Sims, O’Bannon, Scully, and Olian 1994; Harrison, Price, and Bell 1998). The establishment of such team-specific experience needs time and commitment. It can become a competitive advantage as tacit and social knowledge is generated, which can be acquired by personal interaction only (Kor 2003). The argumentation is in line with results from Eisenhardt and Schoonhoven (1990) showing a positive relationship between past shared work experience of the founders and revenues of entrepreneurial firms. As we could not find appropriate empirical evidence within the literature, we strongly suggest future studies focus on the effects of acquaintances among the ETMT.

Firm-specific experience is the knowledge about a firm's initial business purpose, the historical business premises, and strategic business development. Such information and experience is best mirrored by founders and, therefore, it is examined in the "Influence of team demographics" section proxied by founders who are part of the ETMT. Experience with VCs was only once used as a control variable in the sample (Busenitz, Fiet, and Moesel 2005). More intense focus on funding experiences among the ETMT is therefore suggested for further studies.

\subsection{Summary of Findings}

The discussed and relevant significant results from the quantitative models of the sample papers were aggregated and integrated into groups of independent variables in summary tables in order to give an overview of ETMT research outcomes over a period of 20 years. These tables should support further 
research attempts by providing suggestions for testable models via common findings, contradictions, and open research gaps. All relevant clearly significant direct relationships of independent to dependent variables are presented in Table 2. Moreover, interaction effects and control variables are presented in the Appendix in Table 4 and Table 5. The selected variables are limited to a team-specific context due to the focus of this paper. While collecting significant relationships, whenever possible, the most complex and comprehensive models with the highest explanatory power were used to avoid wrong interpretations due to lacking variables controlling for other influences.

The summary tables do not classify for differences in the detailed research methodology or the underlying samples, such as focus on certain industries, sample size, regression methods, or characteristics of survey participants. The main purpose of the summary tables is to give an overview of used variables with proven impact, distribution and focus of research activities, significant relationship between variables, contradictory findings, common findings, and research gaps. Every mark (+ or -) indicates the result of one single paper. If a paper contains more than one model showing similar significant relationships, the relationship is counted once for each paper only if there is no contradiction. Different marks in one cell represent contradictory findings, similar marks represent common findings.

\section{Discussion and Conclusion}

The integrated and synthesized relationships between team variables and a new venture's growth and the ability to raise capital derived from the underlying sample lead to five major influencing clusters. These clusters are supposed to offer an integrated view on today's state of the research area and are open for amendments and contributions of future research findings. Demographic and experience factors define what kind of skills and knowledge a manager brings into the ETMT and how upcoming tasks and challenges are mastered. The type of leadership within an ETMT can influence the atmosphere and motivation of team members. Team dynamics are of major relevance, as they are a main determinant of a team's productivity, frictions, and motivation. While these clusters affect a new venture's growth and funding directly, turnover of team members is mostly a result of an imperfect setup (e.g., Ucbasaran, Lockett, Wright, and Westhead 2003). Altogether, the results confirm the validity of underlying theories with focus on human and social capital.

\subsection{Success and Risk Factors for Entrepreneurial Teams}

Summarizing the findings, several deductions can be made leading to the major factors influencing determinants of success and failure for an ETMT to gain superior growth and to raise necessary capital. The by far most frequently used performance variable is a firm's sales growth, followed by employee growth and asset growth. The size of the team, the level of education, and prior work experience contribute to growth by adding to the capabilities available to the firm. A pursued strategy and cooperation matching existing experience and knowledge within the team and giving the opportunity to leverage such skills supports growth even more. Experience in the same industry and familiarity with founding processes as it comes with founding experience contributes to growth, although there are minor contradictory results for industry experience. The same applies to founders still being members of the ETMT, as they are most cognizant of the initial business purpose and strategy, combined with strong intrinsic motivation.

Heterogeneity can facilitate growth in general or when different functional skills and industry backgrounds complement each other to a powerful and skilled force. However, there is still a risk for frictions and conflict emerging from diverse perspectives, especially in combination with high novelty of products and services. Moreover, empowering and transformational leadership styles are an appropriate way in combination with dynamic environments. It releases forces, potency, and flexibility of individual qualifications necessary to deal with external challenges. As a result, negative effects apply to directive and transactional leadership styles in dynamic environments.

The addition of new team members can help in handling complexity in dynamic environments, while departure subducts necessary capabilities from the firm. Cohesion within a team and cognitive conflict strongly lead to a friendly atmosphere, higher productivity, and less friction, as well as rational problem solving and decision-making necessary for growth. Affective conflict proceeds on an emotional and personal level and thus does not 
Table 2: Summary of significant direct relationships among the sample papers

\begin{tabular}{|c|c|c|c|c|c|c|c|}
\hline \multirow[b]{2}{*}{$\begin{array}{l}\text { Independent } \\
\text { variables }\end{array}$} & \multicolumn{3}{|c|}{ Dependent variable: growth } & \multicolumn{4}{|c|}{ Dependent variable: funding } \\
\hline & $\begin{array}{l}\text { Sales } \\
\text { growth }\end{array}$ & $\begin{array}{l}\text { Employee } \\
\text { growth }\end{array}$ & $\begin{array}{l}\text { Growth index } \\
\text { (sales + employee } \\
\text { growth) }\end{array}$ & $\begin{array}{l}\text { VC fund- } \\
\text { ing }\end{array}$ & $\begin{array}{l}\text { Going public } \\
\text { via IPO }\end{array}$ & $\begin{array}{l}\text { Valuation of } \\
\text { start-up before } \\
\text { VC funding }\end{array}$ & PE financing \\
\hline \multicolumn{8}{|l|}{ Demographics } \\
\hline Team size & + & + & + & + & + & & \\
\hline $\begin{array}{l}\text { Number of founders left in } \\
\text { ETMT }\end{array}$ & + & & & & & & \\
\hline Education of founders & & +++ & - & & & & + \\
\hline Direct or indirect tie to VC & & & & + & & & \\
\hline $\begin{array}{l}\text { Presence of minority } \\
\text { groups or males }\end{array}$ & & + & & & & & \\
\hline Heterogeneity in general & + & & - & & & & \\
\hline $\begin{array}{l}\text { Heterogeneity in years of } \\
\text { industry experience }\end{array}$ & + & & & & & & \\
\hline $\begin{array}{l}\text { Heterogeneity in func- } \\
\text { tional background }\end{array}$ & + & & & + & $-1 /+$ & & \\
\hline $\begin{array}{l}\text { Heterogeneity in educa- } \\
\text { tion level }\end{array}$ & $-1+$ & & & & + & & \\
\hline $\begin{array}{l}\text { Cognitive comprehensive- } \\
\text { ness of team members }\end{array}$ & + & & & & & & \\
\hline \multicolumn{8}{|l|}{ Leadership } \\
\hline Transactional & + & & + & & & & \\
\hline Directive & & + & + & & & & \\
\hline Transformational & - & & $-1+2$ & & & & \\
\hline Empowering & & - & $-/+5$ & & & & \\
\hline \multicolumn{8}{|l|}{ Turnover } \\
\hline $\begin{array}{l}\text { Management turnover: } \\
\text { departures }\end{array}$ & & & & + & -- & & \\
\hline $\begin{array}{l}\text { Founder turnover: depar- } \\
\text { tures }\end{array}$ & & - & & & + & & \\
\hline $\begin{array}{l}\text { Management turnover: } \\
\text { additions }\end{array}$ & & & & - & ++ & & \\
\hline \multicolumn{8}{|l|}{ Dynamics } \\
\hline Cohesion & +++ & & & & & & \\
\hline Cognitive conflict & ++ & & & & & & \\
\hline Affective conflict & --- & & & & & & \\
\hline
\end{tabular}

${ }^{1}$ Refers to the founding team

${ }^{2}$ Negative for vertical leadership (top-down leadership by an individual), positive for shared leadership (collaborative leadership in a team) 
Table 2 continued: Summary of significant direct relationships among the sample papers

\begin{tabular}{|c|c|c|c|c|c|c|c|}
\hline \multirow[b]{2}{*}{$\begin{array}{l}\text { Independent } \\
\text { variables }\end{array}$} & \multicolumn{3}{|c|}{ Dependent variable: growth } & \multicolumn{4}{|c|}{ Dependent variable: funding } \\
\hline & $\begin{array}{l}\text { Sales } \\
\text { growth }\end{array}$ & $\begin{array}{l}\text { Employee } \\
\text { growth }\end{array}$ & $\begin{array}{l}\text { Growth index } \\
\text { (sales + employee } \\
\text { growth) }\end{array}$ & $\begin{array}{l}\text { VC fund- } \\
\text { ing }\end{array}$ & $\begin{array}{l}\text { Going public } \\
\text { via IPO }\end{array}$ & $\begin{array}{l}\text { Valuation of } \\
\text { start-up before } \\
\text { VC funding }\end{array}$ & PE financing \\
\hline \multicolumn{8}{|l|}{ Experience } \\
\hline $\begin{array}{l}\text { Work experience of foun- } \\
\text { ders and management }\end{array}$ & + & + & + & ++ & + & & \\
\hline Founding experience & & + & + & - & + & + & \\
\hline $\begin{array}{l}\text { Experience in same indus- } \\
\text { try }\end{array}$ & $-1+$ & ++ & & & + & & \\
\hline $\begin{array}{l}\text { Number of overlapping } \\
\text { prior companies of foun- } \\
\text { ders }\end{array}$ & & & & & + & & \\
\hline $\begin{array}{l}\text { Breadth of networks } \\
\text { among founders and } \\
\text { management }\end{array}$ & & & & + & + & + & \\
\hline
\end{tabular}

contribute to efficient problem solving in favor of the new venture. The presence of ethnic immigrants, although expected to contribute to diversity, has a negative influence on growth in combination with relatively old teams. Instead, the presence of minority groups and a high rate of male ETMT members facilitate growth positively.

The probability of funding (through VCs, PE firms, or going public) and firm valuation all facilitate a new venture's ability to raise capital. A team's education and work experience are important quality factors for investors evaluating a new venture. Especially shared work experience in the same prior company affiliations signals professional experience and knowledge about team members leading to less friction during team-building phases. Additionally, industry experience facilitates IPOs.

Team members heterogeneous in their functional background are expected to provide a more diverse set of skills, knowledge, and experience contributing positively to a firm's outcomes and thus increasing funding probability. Nonetheless, the findings concerning the impact on going public for founding teams are contradictory. Individuals within the team with founding experience and especially with high returns of prior start-ups have already proven entrepreneurial abilities and motivation, leading to an easier access to capital, although there are detrimental effects specifically for VC funding. Further positive evaluations result when team members have direct or indirect ties to VCs or can utilize an extensive network, indicating richness in social capital.

Finally, investors are more likely to fund a new venture when founders are replaced by more experienced and skilled external managers after a while. However, departure of managers in general signals instability of the team and reduces the probability of receiving funds.

\subsection{Suggestions for Further Research}

The current state of research in the field of ETMTs and their influence on a firm's growth and funding abilities is situated somewhere between still developing and maturity. This section attempts to point out issues that still need to be considered and suggestions for further research worthwhile to dig deeper into, gain new findings, and expand current research.

In the field of demographic factors, further investigation of an entrepreneur's social and financial background might be of interest. The social status of a founder, his or her financial leeway, and the wealth $\mathrm{s} / \mathrm{he}$ possesses is expected to influence behavior, social ties, cognitive base, and motivation. High wealth is suggested to reduce a founder's commitment as $\mathrm{s} /$ he is not financially dependent on the new venture, extending a study by Hvide and Moen (2010). Several parallel firms in the portfolio of an entrepreneur can lead to a lack of awareness and attention to a single venture. The money or private wealth of a founder invested in the new ven- 
ture should not be measured in absolute terms, but in relative terms to his or her total wealth as a more precise proxy for commitment due to differences in the financial leeway of individuals. Such research can contribute to a set of hypotheses and theories summarized under the concept of entrepreneurial capital as mentioned by Erikson (2002).

Team-internal dynamics are another area offering further research potential. Prior research fails to include a power dimension into the observation of team dynamics. It can be of interest to investigate the influence of the distribution of power among the team members and how it influences team processes, turnover, firm outcomes, and the willingness to hire and delegate, following Finkelstein (1992). The individual motivation of founders points in a similar direction, as differences (e.g., financial, carrier, self-fulfillment) might influence firm outcomes, team-internal moods and atmosphere when such motivations become public. Furthermore, the origin of the acquaintance of team members with each other can be a critical success factor of a team's efficiency (Parise and Rollag 2010). Prior shared professional work experience is expected to be more beneficial to efficiently working as a team compared to a prior friendship relation, which instead is expected to be more beneficial to social interactions within the team. This distinction was already made by Franke, Gruber, Harhoff, and Henkel (2008).

One might become even more specific and elaborate the origin of acquaintance among the team in more detail. Vanaelst, Clarysse, Wright, Lockett, Moray, and S'Jegers (2006) for instance discussed the team heterogeneity of academic spinouts and Shane and Stuart (2002) focused on the effects of social capital within university start-ups. Such research can be advanced by analyzing the ability to leverage the universities' knowledge resources into attractive business cases. The same specific focusing can be applied to family businesses where prior relationships are not just work- or friendship-based. Instead the acquaintance type can be expected to be even more intense and cohesive, social bonds can be leveraged, and the threshold to break apart is comparably higher (e.g., Ensley and Pearson 2005).

External influences and dynamics are another field where further investigation is suggested. It can be of interest to analyze how such influences from investors, banks, boards, advisors, and networks, etc. influence an ETMT and thus distort findings of prior research. Another question could ask how networks and social ties of individuals contribute to team turnover (e.g., Ucbasaran, Lockett, Wright, and Westhead 2003; Witt 2004). The opportunities (e.g., new job offers, other venture foundings) available to the individuals on the team are expected to correlate with social ties and might increase member turnover.

One stakeholder is of special interest as VCs often cooperate with new ventures due to their novelty, risk, and uncertainty. While some facets of dynamics between VCs and ETMTs were already investigated, further research is necessary to not only focus on funding decisions but to also emphasize the ongoing relationships after funding (e.g., Busenitz, Fiet, and Moesel 2004). The room available to steer and manage the expectations of VCs, as well as conflict handling in the sometimes challenging relation between entrepreneurial visions and investor's exit strategies, offers potential for a better understanding of such processes (Busenitz, Moesel, Fiet, and Barney 1997).

Furthermore, motivated by Akgün and Lynn (2002) and Hayes, Oyer, and Schaefer (2004), the examination of the strengths of team-internal bonds and cohesion might be useful in order to measure stability of teams in case of stress and setbacks. The stability of leaders within the team points in a similar direction. For example, VCs and other stakeholders can urge changes in the ETMT in order to professionalize a venture's management (Chandler, Honig, and Wiklund 2005). Leadership research on firm outcomes in an entrepreneurial team context in general is underrepresented. Development of leaders within an ETMT, team-internal power struggles, and the influence of leadership styles on team dynamics and individuals are expected to offer great potential for further analysis.

Raising capital in contrast to growth measures has received minor attention in prior research. While the relationship to VCs and the factors influencing positive funding decisions were discussed in some papers already, the influencing factors of going public are underrepresented. The impact of experience, demographics, and turnover on going public was tested already (e.g., Beckman, Burton, and O'Reilly 2007; Beckman and Burton 2008; Shane and Stuart 2002), while team dynamics and leadership factors were neglected so far and could reveal important insights into IPO research.

Further research might be executed on the tenure an original founder's business idea stays in the firm 
represented by, e.g., strategies or visions especially when all founders have left the top management. Deviation from this original business purpose is expected to affect a firm's performance (e.g., Baron and Hannan 2002). Apart from the introduction or new combination of independent and dependent variables the analysis of interacting effects has revealed several findings in prior research which indicates the complexity of relationships in this research field (e.g., Packalen 2007). While this statement holds for growth variables, funding variables were seldom tested on interacting effects. It is suggested to pursue such analysis of interaction effects in order to deepen insights and clarify details and context of known influences. The same criticism applies to the neglected analysis of indirect effects via mediating variables. Further research for such more complex causal models is expected to again result in more high-quality and sophisticated findings. Overall, the research outcomes already explored and the suggestions for further research aiming at identified research gaps contribute to understanding success factors, costs, and potential problems of team assembly and configuration in an entrepreneurial context.

\section{Appendix A: Sampling process}

1. Search was conducted on the following databases: EBSCO Business Source Premier and EconLit, Web of Science SSCI (Social Science Citation Index).

2. Search is limited to papers published in scholarly journals which were peer reviewed only in order to ensure substantive quality and relevance for practical research.

3. Primary keywords (where * represents any given number of additional characters):

a. Papers are required to contain at least one of the following primary keywords in their title or abstract: "top management*", "TMT", "*team*", "upper echelon*".

b. Papers are required to contain at least one of the following primary keywords in their title or abstract: "entrepreneur*", "new venture*", "start up"”, "start-up*".

c. Papers are required to contain at least one of the following primary keywords in their title or abstract: "*success"”, "*performance", "IPO", "initial public offering", "VC", "venture capital".
4. Papers are required to contain at least one of the following 29 additional keywords in their title or abstract: "power", "influence", "composition", "collaboration", "teamwork", "atmosphere", "mood*", "trust", "motivation", "delegation", "diversit*", "heterogen*", "relationship*", "fluctuation", "turnover", "decision making”, "decision-making", "gender", "age", "academic background", "education", "nationality", "experience”, "knowledge”, "development", "young", "diversif*”, "behaviour*", "behavior".

5. To ensure empirical content, papers are required to contain at least one of the following seven methodological keywords in their title or abstract: "data", "empirical", "test", "statistical", "evidence", "finding*", "result*".

6. Results are consolidated from all databases and duplicates eliminated.

7. Papers published in journals, which are not frequently cited $^{1}$, are excluded due to insufficient relevance and low impact on scientific value add.

8. Case studies and qualitative research are excluded to ensure the ability to compare and integrate quantitative models and to apply a systematic coding scheme.

9. Relevance of the dependent variable in quantitative models is ensured (e.g., growth, VC funding, IPO).

10. Relevance of the independent variable in the quantitative model is ensured (e.g., TMT characteristics, academic background, experience, team size, team heterogeneity).

11. All abstracts of the remaining sample are screened for substantive context (i.e. discussion of questions related to the literature review focus) and empirical relevance (i.e. mention of statistical analysis and significant results).

12. All remaining papers in their entirety are screened for substantive context (i.e. discussion of questions related to the literature review focus) and empirical relevance (i.e. men-

\footnotetext{
${ }^{1}$ Indicator for high-quality journals as requested by Cooper 1994; citation-index measured via 5-year JCR impact factor; data from Web of Knowledge JCR as of 06.05.2010; journals with 5-year JCR impact factor below 2.0 were excluded; for calculation details see: http://admin-apps.isiknowledge.com/ JCR/help/h_impfact.htm
} 
tion of statistical analysis and significant results).

13. All references of remaining papers are screened to identify further relevant literature; additional recommendations by one of the anonymous reviewers were also included.

Table 3: Overview of auxiliary sample papers

\begin{tabular}{|c|c|c|c|}
\hline Year & Title & Author(s) & Published in \\
\hline 2007 & $\begin{array}{l}\text { Small worlds, infinite possibilities? How social networks affect } \\
\text { entrepreneurial team formation and search }\end{array}$ & $\begin{array}{l}\text { Aldrich, Howard E.; Kim, } \\
\text { Phillip H. }\end{array}$ & Strategic Entrepreneurship Journal \\
\hline 2007 & Resources, capabilities and entrepreneurial perceptions & $\begin{array}{l}\text { Kor, Yasemin Y.; Ma- } \\
\text { honey, Joseph T.; Mi- } \\
\text { chael, Steven C. }\end{array}$ & Journal of Management Studies \\
\hline 2007 & $\begin{array}{l}\text { Collective cognition: when entrepreneurial teams, not individu- } \\
\text { als, make decisions }\end{array}$ & West III, G. Page & Entrepreneurship: Theory \& Practice \\
\hline 2007 & $\begin{array}{l}\text { Complementing capital: the role of status, demographic fea- } \\
\text { tures, and social capital in founding teams' abilities to obtain } \\
\text { resources }\end{array}$ & Packalen, Kelley A. & Entrepreneurship: Theory \& Practice \\
\hline 2006 & $\begin{array}{l}\text { Effects of team inputs and intrateam processes on perceptions } \\
\text { of team viability and member satisfaction in nascent ventures }\end{array}$ & $\begin{array}{l}\text { Foo, Maw-Der; Sin, } \\
\text { Hock-Peng; Yiong, Lee- } \\
\text { Pen }\end{array}$ & Strategic Management Journal \\
\hline 2006 & $\begin{array}{l}\text { Entrepreneurial team development in academic spinouts: an } \\
\text { examination of team heterogeneity }\end{array}$ & $\begin{array}{l}\text { Vanaelst, Iris; Clarysse, } \\
\text { Bart; Wright, Mike; } \\
\text { Lockett, Andy; Moray, } \\
\text { Nathalie; S'Jegers, Ro- } \\
\text { sette }\end{array}$ & Entrepreneurship: Theory \& Practice \\
\hline 2005 & $\begin{array}{l}\text { A quantitative content analysis of the characteristics of rapid- } \\
\text { growth firms and their founders }\end{array}$ & $\begin{array}{l}\text { Barringer, Bruce R.; } \\
\text { Jones, Foard F.; Neu- } \\
\text { baum, Donald O. }\end{array}$ & Journal of Business Venturing \\
\hline 2005 & New venture evolution and managerial capabilities & $\begin{array}{l}\text { Boeker, Warren; Wilt- } \\
\text { bank, Robert }\end{array}$ & Organization Science \\
\hline 2005 & $\begin{array}{l}\text { An exploratory comparison of the behavioral dynamics of top } \\
\text { management teams in family and nonfamily new ventures: } \\
\text { cohesion, conflict, potency, and consensus }\end{array}$ & $\begin{array}{l}\text { Ensley, Michael D.; } \\
\text { Pearson, Allison W. }\end{array}$ & Entrepreneurship: Theory \& Practice \\
\hline 2004 & $\begin{array}{l}\text { Characteristics, contracts, and actions: evidence from venture } \\
\text { capitalist analyses }\end{array}$ & $\begin{array}{l}\text { Kaplan, Steven N.; } \\
\text { Strömberg, Per }\end{array}$ & Journal of Finance \\
\hline 2004 & Entrepreneurs' networks and the success of start-ups & Witt, Peter & $\begin{array}{l}\text { Entrepreneurship \& Regional Devel- } \\
\text { opment }\end{array}$ \\
\hline 2003 & $\begin{array}{l}\text { Top management team process, shared leadership, and new } \\
\text { venture performance: a theoretical model and research agenda }\end{array}$ & $\begin{array}{l}\text { Ensley, Michael D.; } \\
\text { Pearson, Allison W.; } \\
\text { Pearce, Craig L. }\end{array}$ & $\begin{array}{l}\text { Human Resource Management Re- } \\
\text { view }\end{array}$ \\
\hline 2003 & $\begin{array}{l}\text { The risks of autonomy: empirical evidence for the necessity of a } \\
\text { balance management in promoting organizational innovative- } \\
\text { ness }\end{array}$ & $\begin{array}{l}\text { Gebert, Diether; Boerner, } \\
\text { Sabine; Lanwehr, Ralf }\end{array}$ & , Creativity \& Innovation Management \\
\hline 2003 & Transactors, transformers and beyond & $\begin{array}{l}\text { Pearce, Craig L.; Sims, } \\
\text { Henry P.; Cox, Jonathan } \\
\text { F.; Ball, Gail; Schnell, } \\
\text { Eugene; Smith, Ken A.; } \\
\text { Trevino, Linda }\end{array}$ & Journal of Management Development \\
\hline 2003 & $\begin{array}{l}\text { Entrepreneurial founder teams: factors associated with member } \\
\text { entry and exit }\end{array}$ & $\begin{array}{l}\text { Ucbasaran, Deniz; } \\
\text { Lockett, Andy; Wright, } \\
\text { Mike; Westhead, Paul }\end{array}$ & Entrepreneurship: Theory \& Practice \\
\hline
\end{tabular}

\section{Appendix B: Auxiliary sample}

This auxiliary sample comprises of 68 papers which did not meet all requirements of the sampling procedure. However, they are still worth to mention in order to give the reader the opportunity to become familiar with the underlying subject in more detail and refer to further sources beyond this literature review. 


\section{Table 3 continued: Overview of auxiliary sample papers}

\begin{tabular}{|c|c|c|c|}
\hline Year & Title & Author(s) & Published in \\
\hline 2002 & $\begin{array}{l}\text { Confidence at the group level of analysis: a longitudinal investi- } \\
\text { gation of the relationship between potency and team effective- } \\
\text { ness }\end{array}$ & $\begin{array}{l}\text { Pearce, Craig L.; Galla- } \\
\text { gher, Cynthia A.; Ensley, } \\
\text { Michael D. }\end{array}$ & $\begin{array}{l}\text { Journal of Occupational and Organ- } \\
\text { izational Psychology }\end{array}$ \\
\hline 2002 & $\begin{array}{l}\text { The antecedents and consequences of group potency: a longitu- } \\
\text { dinal investigation of newly formed work groups }\end{array}$ & $\begin{array}{l}\text { Meglino, Bruce M.; } \\
\text { Lester, Scott W.; } \\
\text { Korsgaard, M. Audrey }\end{array}$ & Academy of Management Journal \\
\hline 2002 & Social capital: prospects for a new concept & $\begin{array}{l}\text { Adler, Paul S.; Kwon, } \\
\text { Seok-Woo }\end{array}$ & Academy of Management Review \\
\hline 2002 & $\begin{array}{l}\text { Venture capital and the professionalization of start-up firms: } \\
\text { empirical evidence }\end{array}$ & \multicolumn{2}{|c|}{$\begin{array}{l}\text { Hellmann, Thomas; Puri, Journal of Finance } \\
\text { Manju }\end{array}$} \\
\hline 2001 & $\begin{array}{l}\text { Exploring antecedents to top management team conflict: the } \\
\text { importance of behavioral integration }\end{array}$ & $\begin{array}{l}\text { Mooney, Ann C.; Son- } \\
\text { nenfeld, Jeffrey }\end{array}$ & Academy of Management Proceedings \\
\hline 2001 & $\begin{array}{l}\text { Qualifications and turnover of managers and venture capital- } \\
\text { financed firm performance: an empirical study of German } \\
\text { venture capital-investments }\end{array}$ & $\begin{array}{l}\text { Schefczyk, Michael; } \\
\text { Gerpott, Torsten J. }\end{array}$ & Journal of Business Venturing \\
\hline 2001 & $\begin{array}{l}\text { Voluntary turnover, social capital, and organizational perform- } \\
\text { ance }\end{array}$ & $\begin{array}{l}\text { Dess, Gregory G.; Shaw, } \\
\text { Jason D. }\end{array}$ & Academy of Management Review \\
\hline 2001 & The managerial rents model: theory and empirical analysis & $\begin{array}{l}\text { Castanias, Richard P.; } \\
\text { Helfat, Constance E. }\end{array}$ & Journal of Management \\
\hline 2000 & $\begin{array}{l}\text { A case for comparative entrepreneurship: assessing the rele- } \\
\text { vance of culture }\end{array}$ & $\begin{array}{l}\text { Thomas, Anisya S.; } \\
\text { Mueller, Stephen L. }\end{array}$ & $\begin{array}{l}\text { Journal of International Business } \\
\text { Studies }\end{array}$ \\
\hline 2000 & $\begin{array}{l}\text { Penrose's resource-based approach: the process and product of } \\
\text { research creativity }\end{array}$ & $\begin{array}{l}\text { Kor, Yasemin Y.; Ma- } \\
\text { honey, Joseph T. }\end{array}$ & Journal of Management Studies \\
\hline 2000 & $\begin{array}{l}\text { The effects of development on migration: theoretical issues and } \\
\text { new empirical evidence }\end{array}$ & $\begin{array}{l}\text { Vogler, Michael; Rotte, } \\
\text { Ralph }\end{array}$ & Journal of Population Economics \\
\hline 1999 & $\begin{array}{l}\text { Making use of difference: diversity, debate, and decision com- } \\
\text { prehensiveness in top management teams }\end{array}$ & $\begin{array}{l}\text { Simons, Tony; Pelled, } \\
\text { Lisa Hope; Smith, Ken A. }\end{array}$ & Academy of Management Journal \\
\hline 1999 & $\begin{array}{l}\text { Wachstumsdeterminanten junger innovativer Unternehmen: } \\
\text { Empirische Ergebnisse für West-Deutschland. (Growth Deter- } \\
\text { minants of Young Innovative Firms: Empirical Results for West } \\
\text { Germany. With English summary.) }\end{array}$ & $\begin{array}{l}\text { Almus, Matthias; Nerlin- } \\
\text { ger, Eric }\end{array}$ & $\begin{array}{l}\text { Jahrbücher für Nationalökonomie } \\
\text { und Statistik }\end{array}$ \\
\hline 1998 & $\begin{array}{l}\text { Beyond relational demography: time and the effects of surface- } \\
\text { and deep-level diversity on work group cohesion }\end{array}$ & $\begin{array}{l}\text { Harrison, David A.; Price, } \\
\text { Kenneth H.; Bell, Myrtle } \\
\text { P. }\end{array}$ & ,Academy of Management Journal \\
\hline 1998 & $\begin{array}{l}\text { Cognitive diversity among upper-echelon executives: implica- } \\
\text { tions for strategic decision processes }\end{array}$ & $\begin{array}{l}\text { Miller, C. Chet; Burke, } \\
\text { Linda M.; Glick, William } \\
\text { H. }\end{array}$ & Strategic Management Journal \\
\hline 1997 & $\begin{array}{l}\text { Survival of the fittest? Entrepreneurial human capital and the } \\
\text { persistence of underperforming firms }\end{array}$ & $\begin{array}{l}\text { Gimeno, Javier; Folta, } \\
\text { Timothy B.; Cooper, } \\
\text { Arnold C.; Woo, Carolyn } \\
\text { Y. }\end{array}$ & Administrative Science Quarterly \\
\hline 1997 & $\begin{array}{l}\text { Executive migration and strategic change: the effect of top } \\
\text { manager movement on product-market entry }\end{array}$ & Boeker, Warren & Administrative Science Quarterly \\
\hline 1997 & $\begin{array}{l}\text { A comparative study of values among Chinese and U.S. entre- } \\
\text { preneurs: pragmatic convergence between contrasting cultures }\end{array}$ & Holt, David H. & Journal of Business Venturing \\
\hline 1997 & $\begin{array}{l}\text { The effects of top management team size and interaction norms } \\
\text { on cognitive and affective conflict }\end{array}$ & $\begin{array}{l}\text { Amason, Allen C.; Sapi- } \\
\text { enza, Harry J. }\end{array}$ & Journal of Management \\
\hline 1997 & Specificity of CEO human capital and compensation & $\begin{array}{l}\text { Harris, Dawn; Helfat, } \\
\text { Constance }\end{array}$ & Strategic Management Journal \\
\hline
\end{tabular}




\section{Table 3 continued: Overview of auxiliary sample papers}

\begin{tabular}{|c|c|c|c|}
\hline Year & Title & Author(s) & Published in \\
\hline 1996 & $\begin{array}{l}\text { Distinguishing the effects of functional and dysfunctional con- } \\
\text { flict on strategic decision making: resolving a paradox for top } \\
\text { management teams }\end{array}$ & Amason, Allen C. & Academy of Management Journal \\
\hline 1996 & Social structural characteristics of psychological empowerment & Spreitzer, Gretchen M. & Academy of Management Journal \\
\hline 1996 & The initial succession: a contingency model of founder tenure & $\begin{array}{l}\text { Rubenson, George C.; } \\
\text { Gupta, Anil K. }\end{array}$ & Entrepreneurship: Theory \& Practice \\
\hline 1996 & $\begin{array}{l}\text { Demographic diversity, conflict, and work group outcomes: an } \\
\text { intervening process theory }\end{array}$ & Pelled, Lisa Hope & Organization Science \\
\hline 1995 & $\begin{array}{l}\text { A multimethod examination of the benefits and detriments of } \\
\text { intragroup conflict }\end{array}$ & Jehn, Karen A. & Administrative Science Quarterly \\
\hline 1995 & $\begin{array}{l}\text { Two investigations of the relationships among group goals, goal } \\
\text { commitment, cohesion, and performance }\end{array}$ & $\begin{array}{l}\text { Klein, Howard J.; Mul- } \\
\text { vey, Paul W. }\end{array}$ & $\begin{array}{l}\text { Organizational Behavior and Human } \\
\text { Decision Processes }\end{array}$ \\
\hline 1995 & $\begin{array}{l}\text { Employment change in independent owner-managed high- } \\
\text { technology firms in Great Britain }\end{array}$ & $\begin{array}{l}\text { Westhead, Paul; Cowling } \\
\text { Marc }\end{array}$ & ,Small Business Economics \\
\hline 1994 & $\begin{array}{l}\text { Top management team demography and process: the role of } \\
\text { social integration and communication }\end{array}$ & $\begin{array}{l}\text { Smith, Ken G.; Smith, } \\
\text { Ken A.; Sims, Henry P.; } \\
\text { O'Bannon, Douglas P.; } \\
\text { Scully, Judith A.; Olian, } \\
\text { Judy D. }\end{array}$ & Administrative Science Quarterly \\
\hline 1994 & $\begin{array}{l}\text { The impact of perceived group success - failure on motivational } \\
\text { beliefs and attitudes: a causal model }\end{array}$ & $\begin{array}{l}\text { Riggs, Matt L.; Knight, } \\
\text { Patrick A. }\end{array}$ & Journal of Applied Psychology \\
\hline 1993 & Configurational approaches to organizational analysis & $\begin{array}{l}\text { Meyer, Alan D.; Tsui, } \\
\text { Anne S.; Hinings, C. R. }\end{array}$ & Academy of Management Journal \\
\hline 1993 & The stages of team venture formation: a decision-making model & $\begin{array}{l}\text { Kamm, Judith B., Nurick } \\
\text { Aaron J. }\end{array}$ & ,Entrepreneurship: Theory \& Practice \\
\hline 1992 & Survival chances of newly founded business organizations & $\begin{array}{l}\text { Brüderl, Josef; Preisen- } \\
\text { dörfer, Peter; Ziegler, } \\
\text { Rolf }\end{array}$ & American Sociological Review \\
\hline 1992 & $\begin{array}{l}\text { Top management team demography and corporate strategic } \\
\text { change }\end{array}$ & $\begin{array}{l}\text { Wiersema, Margarethe } \\
\text { F.; Bantel, Karen A. }\end{array}$ & Academy of Management Journal \\
\hline 1992 & $\begin{array}{l}\text { Consequences of group composition for the interpersonal dy- } \\
\text { namics of strategic issue processing }\end{array}$ & Jackson, Susan E. & Advances in Strategic Management \\
\hline 1992 & $\begin{array}{l}\text { Does culture endure, or is it malleable? Issues for entrepreneu- } \\
\text { rial economic development }\end{array}$ & $\begin{array}{l}\text { McGrath, Rita Gunther; } \\
\text { MacMillan, Ian C.; Yang, } \\
\text { Elena Ai-Yuan; Tsai, } \\
\text { William }\end{array}$ & Journal of Business Venturing \\
\hline 1992 & Thinking about entrepreneurship: a fuzzy set approach & Hornaday, Robert W. & $\begin{array}{l}\text { Journal of Small Business Manage- } \\
\text { ment }\end{array}$ \\
\hline 1991 & $\begin{array}{l}\text { Entrepreneurs in high technology - Lessons from MIT and } \\
\text { beyond }\end{array}$ & Roberts, Edward Baer & Oxford University Press \\
\hline 1990 & $\begin{array}{l}\text { What are we talking about when we talk about entrepreneur- } \\
\text { ship? }\end{array}$ & Gartner, William B. & Journal of Business Venturing \\
\hline 1990 & Perceived cohesion: a conceptual and empirical examination & $\begin{array}{l}\text { Bollen, Kenneth A.; } \\
\text { Hoyle, Rick H. }\end{array}$ & Social Forces \\
\hline 1990 & $\begin{array}{l}\text { Top management team group factors, consensus, and firm } \\
\text { performance }\end{array}$ & Priem, Richard L. & Strategic Management Journal \\
\hline 1989 & Top management group heterogeneity and firm performance & Murray, Alan I. & Strategic Management Journal \\
\hline 1989 & Work group demography, social integration, and turnover & $\begin{array}{l}\text { O'Reilly III, Charles A.; } \\
\text { Caldwell, David F.; } \\
\text { Barnett, William P. }\end{array}$ & Administrative Science Quarterly \\
\hline
\end{tabular}


Table 3 continued: Overview of auxiliary sample papers

\begin{tabular}{|c|c|c|c|}
\hline Year & Title & Author(s) & Published in \\
\hline 1988 & $\begin{array}{l}\text { Attributions and organizational conflict: the mediating role of } \\
\text { apparent sincerity }\end{array}$ & Baron, Robert A. & $\begin{array}{l}\text { Organizational Behavior \& Human } \\
\text { Decision Processes }\end{array}$ \\
\hline 1987 & $\begin{array}{l}\text { Do good or poor performers leave? A meta-analysis of the rela- } \\
\text { tionship between performance and turnover }\end{array}$ & $\begin{array}{l}\text { McEvoy, Glenn M.; } \\
\text { Cascio, Wayne F. }\end{array}$ & Academy of Management Journal \\
\hline 1986 & $\begin{array}{l}\text { Group approaches for improving strategic decision making: a } \\
\text { comparative analysis of dialectical inquiry, devil's advocacy, and } \\
\text { consensus }\end{array}$ & $\begin{array}{l}\text { Schweiger, David M.; } \\
\text { Sandberg, William R.; } \\
\text { Ragan, James W. }\end{array}$ & Academy of Management Journal \\
\hline 1985 & Outcomes of role stress: a multisample constructive replication & $\begin{array}{l}\text { Kemery, Edward R.; } \\
\text { Bedeian, Arthur G.; } \\
\text { Mossholder, Kevin W.; } \\
\text { Touliatos, John }\end{array}$ & Academy of Management Journal \\
\hline 1984 & $\begin{array}{l}\text { Upper echelons: the organization as a reflection of its top man- } \\
\text { agers }\end{array}$ & $\begin{array}{l}\text { Hambrick, Donald C.; } \\
\text { Mason, Phyllis A. }\end{array}$ & Academy of Management Review \\
\hline 1984 & $\begin{array}{l}\text { Matching managers to strategies: a review and suggested } \\
\text { framework }\end{array}$ & $\begin{array}{l}\text { Szilagyi, Andrew D.; } \\
\text { Schweiger, David M. }\end{array}$ & Academy of Management Review \\
\hline 1978 & $\begin{array}{l}\text { A social information processing approach to job attitudes and } \\
\text { task design }\end{array}$ & $\begin{array}{l}\text { Salancik, Gerald R.; } \\
\text { Pfeffer, Jeffrey }\end{array}$ & Administrative Science Quarterly \\
\hline 1976 & $\begin{array}{l}\text { A model of task group development in complex organizations } \\
\text { and a strategy of implementation }\end{array}$ & $\begin{array}{l}\text { Heinen, J. Stephen; } \\
\text { Jacobson, Eugene }\end{array}$ & Academy of Management Review \\
\hline 1970 & Age, cohorts and the generation of generations & $\begin{array}{l}\text { Carlsson, Gosta; Karls- } \\
\text { son, Katarina }\end{array}$ & American Sociological Review \\
\hline 1965 & Social structure and organizations & Stinchcombe, A. L. & Rand McNally Publishers \\
\hline 1959 & The theory of the growth of the firm & Penrose, Edith & Blackwell \\
\hline
\end{tabular}

In addition with the sample of this literature review (28 papers) the auxiliary sample adds up to 96 papers and comes to the following descriptive statistics:

\section{Figure 3: Distribution of auxiliary sample papers}

Distribution of the sample papers by publishing date ( $\Sigma 96$ paper)

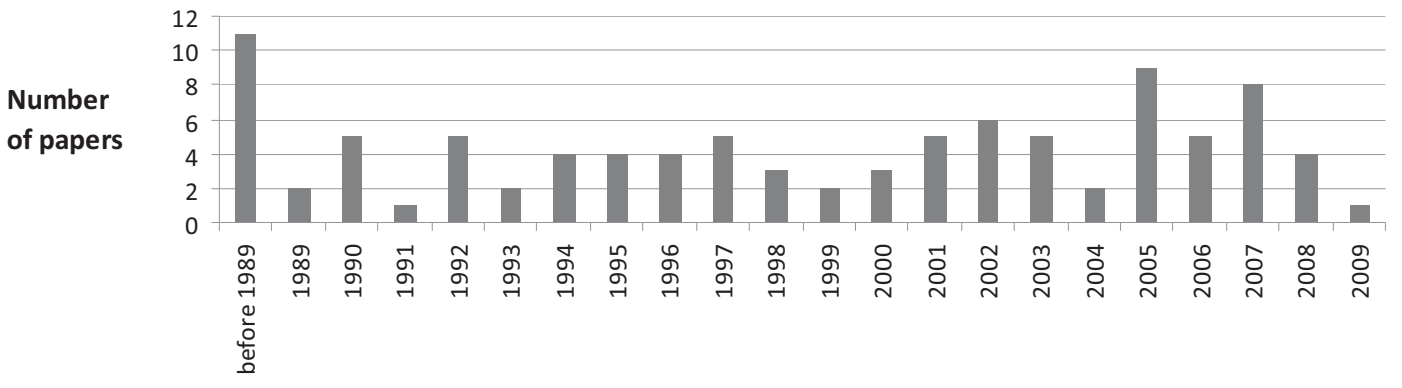

Distribution of the sample papers by publishing date ( $\Sigma 96$ paper)

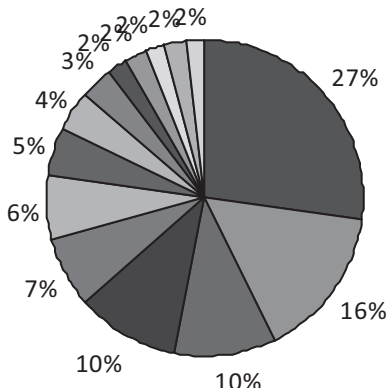

$10 \%$ $\square$ Others

$\square$ Journal of Business Venturing

$\square$ Entrepreneurship Theory \& Practice

$\square$ Academy of Management Journal

$\square$ Strategic Management Journal

$\square$ Adminis trative Science Quarterly

$\square$ Academy of Management Review

$\square$ Organization Science

$\square$ Research Policy

$\square$ Organizational Behavior and Human Decision Processes

$\square$ American Sociological Review

$\square$ Journal of Management

$\square$ Journal of Organizational Behavior

$\square$ Journal of Finance 


\section{Appendix C: Interaction variables}

\section{Table 4: Summary of significant interactions among the sample papers and the direction of the interaction term}

\begin{tabular}{|c|c|c|c|c|c|c|c|c|c|c|c|c|}
\hline $\begin{array}{l}\text { Interaction } \\
\text { variables }\end{array}$ & $\begin{array}{l}\text { Managerial Heterogeneity } \\
\text { or technical in functional } \\
\text { education of background } \\
\text { founders }\end{array}$ & $\begin{array}{l}\text { Experience } \\
\text { in same } \\
\text { industry }\end{array}$ & $\begin{array}{l}\text { Founders } \\
\text { left in } \\
\text { ETMT }\end{array}$ & $\begin{array}{l}\text { Heterogeneity } \\
\text { in age }\end{array}$ & $\begin{array}{l}\text { Heterogeneity } \\
\text { in educational } \\
\text { level }\end{array}$ & $\begin{array}{l}\text { Presence of } \\
\text { ethnic im- } \\
\text { migrants }\end{array}$ & $\begin{array}{l}\text { Network } \\
\text { constraints }\end{array}$ & $\begin{array}{l}\text { Experience- } \\
\text { specific } \\
\text { strategy }\end{array}$ & $\begin{array}{l}\text { Firm: exter- } \\
\text { nal coopera- } \\
\text { tion }\end{array}$ & $\begin{array}{l}\text { Environmental } \\
\text { dynamism }\end{array}$ & $\begin{array}{l}\text { Venture stage } \\
\text { of develop- } \\
\text { ment }\end{array}$ & $\begin{array}{l}\text { Firm } \\
\text { age }\end{array}$ \\
\hline $\begin{array}{l}\text { Heterogeneity in } \\
\text { general }\end{array}$ & & & & & & & & & & $\stackrel{2}{\text { (growth index) }}$ & & \\
\hline $\begin{array}{l}\text { Heterogeneity in } \\
\text { years of industry } \\
\text { experience }\end{array}$ & & & & & & & & & & & & $\begin{array}{c}+ \\
\text { (sales } \\
\text { growth) }\end{array}$ \\
\hline $\begin{array}{l}\text { Leadership transac- } \\
\text { tional }\end{array}$ & & & & & & & & & & $\begin{array}{c}- \\
\text { (sales growth) }\end{array}$ & & \\
\hline $\begin{array}{l}\text { Leadership direc- } \\
\text { tive }\end{array}$ & & & & & & & & & & $\stackrel{-}{\text { (growth index) }}$ & & \\
\hline $\begin{array}{l}\text { Leadership trans- } \\
\text { formational }\end{array}$ & & & & & & & & & & $\stackrel{+}{+}$ & & \\
\hline $\begin{array}{l}\text { Leadership em- } \\
\text { powering }\end{array}$ & & & & & & & & & & $\stackrel{+}{\stackrel{+}{\text { (growth index) }}}$ & & \\
\hline $\begin{array}{l}\text { Management } \\
\text { turnover: additions }\end{array}$ & & & & & & & & & & $\stackrel{+}{\stackrel{+}{(s a l e s} \text { growth) }}$ & $\begin{array}{c}- \\
\text { (sales growth) }\end{array}$ & \\
\hline $\begin{array}{l}\text { Management } \\
\text { turnover: depar- } \\
\text { tures }\end{array}$ & & & & & & & & & & $\begin{array}{c}- \\
\text { (sales growth) }\end{array}$ & $\stackrel{+}{+}$ & \\
\hline Cohesion & & & & & & & $\begin{array}{c}- \\
\text { (sales } \\
\text { growth) }\end{array}$ & & & & & \\
\hline
\end{tabular}

2 hetero. * env.dyn. * empowering leadership: +; hetero. * env.dyn. * directive leadership: -

$\begin{array}{lll}\begin{array}{ll}\text { Variable exists in } \\ \text { Table and is team- }\end{array} & \begin{array}{l}\text { Variable does not } \\ \text { exist in Table and is }\end{array} & \begin{array}{l}\text { Variable does not } \\ \text { exist in Table and }\end{array} \\ & \end{array}$

specific team-specific not team-specific 
Table 4 continued: Summary of significant interactions among the sample papers and the direction of the interaction term

\begin{tabular}{|c|c|c|c|c|c|c|c|c|c|c|c|}
\hline $\begin{array}{l}\text { Interaction } \\
\text { variables }\end{array}$ & $\begin{array}{l}\text { Managerial } \\
\text { or technical } \\
\text { education of } \\
\text { founders }\end{array}$ & $\begin{array}{l}\text { Heterogeneity } \\
\text { in functional } \\
\text { background }\end{array}$ & $\begin{array}{l}\text { Experience } \\
\text { in same } \\
\text { industry }\end{array}$ & $\begin{array}{l}\text { Founders } \\
\text { left in } \\
\text { ETMT }\end{array}$ & $\begin{array}{l}\text { Heterogeneity } \\
\text { in age }\end{array}$ & $\begin{array}{l}\text { Heterogeneity } \\
\text { in educational } \\
\text { level }\end{array}$ & $\begin{array}{ll}\text { Presence of } & \text { Network } \\
\text { ethnic im- } & \text { constraints } \\
\text { migrants } & \end{array}$ & $\begin{array}{l}\text { Experience- } \\
\text { specific } \\
\text { strategy }\end{array}$ & $\begin{array}{ll}\text { Firm: exter- } & \text { Environmental } \\
\text { nal coopera- } & \text { dynamism } \\
\text { tion } & \end{array}$ & $\begin{array}{l}\text { Venture stage } \\
\text { of develop- } \\
\text { ment }\end{array}$ & $\begin{array}{l}\text { Firm } \\
\text { age }\end{array}$ \\
\hline $\begin{array}{l}\text { Work experience of } \\
\text { founders and } \\
\text { management }\end{array}$ & & & $\begin{array}{c}+ \\
\text { (employee } \\
\text { growth) }\end{array}$ & & & & & $\begin{array}{c}+ \\
\text { (sales growth) }\end{array}$ & & & \\
\hline $\begin{array}{l}\text { Experience in same } \\
\text { industry }\end{array}$ & & & & $\begin{array}{c}- \\
\text { (sales } \\
\text { growth) }\end{array}$ & & & & & $\begin{array}{c}+ \\
\text { (sales growth, } \\
\text { technology } \\
\text { coops. only) }\end{array}$ & & \\
\hline $\begin{array}{l}\text { Cooperation spe- } \\
\text { cific functional } \\
\text { experience }\end{array}$ & & & & & & & & & $\stackrel{+}{\stackrel{+}{\text { (sales growth) }}}$ & & \\
\hline $\begin{array}{l}\text { Pre-existing rela- } \\
\text { tionships within } \\
\text { TMT }\end{array}$ & & & & $\begin{array}{l}- \\
\text { (sales } \\
\text { growth) }\end{array}$ & & & & & & & \\
\hline TMT age & & & & & & & $\begin{array}{c}- \\
\text { (sales, em- } \\
\text { ployee, and } \\
\text { asset growth) }\end{array}$ & & & & \\
\hline Industry: internet & $\begin{array}{c}+ \\
\text { (valuation of } \\
\text { start-up } \\
\text { before VC } \\
\text { funding) }\end{array}$ & & & & & & & & & & \\
\hline $\begin{array}{l}\text { Low novelty of } \\
\text { products and } \\
\text { services }\end{array}$ & & & & & $\begin{array}{c}+ \\
\text { (sales growth) }\end{array}$ & & & & & & \\
\hline $\begin{array}{l}\text { High novelty of } \\
\text { products and } \\
\text { services }\end{array}$ & & $\begin{array}{c}- \\
\text { (sales growth) }\end{array}$ & & & $\begin{array}{c}- \\
\text { (sales growth) }\end{array}$ & $\stackrel{-}{\text { (sales growth) }}$ & & & & & \\
\hline
\end{tabular}

${ }^{2}$ hetero. * env.dyn. * empowering leadership: + ; hetero. * env.dyn. * directive leadership: -

$\begin{array}{lll}\begin{array}{l}\text { Variable exists in } \\ \text { Table and is team- }\end{array} & \begin{array}{l}\text { Variable does not } \\ \text { exist in Table and is }\end{array} & \begin{array}{l}\text { Variable does not } \\ \text { exist in Table and is }\end{array} \\ & & \end{array}$

$\begin{array}{lll}\text { exist in Table and is } & \text { exist in Table and } \\ \text { specific } & \text { team-specific } & \text { not team-specific }\end{array}$ 


\section{Appendix D: Control variables}

Table 5: Summary of significant control variables among the sample papers

\begin{tabular}{|c|c|c|c|c|c|c|}
\hline \multirow{2}{*}{$\begin{array}{l}\text { Control } \\
\text { variables }\end{array}$} & \multicolumn{3}{|c|}{ Dependent variable: growth } & \multicolumn{3}{|c|}{ Dependent variable: funding } \\
\hline & $\begin{array}{l}\text { Sales } \\
\text { growth }\end{array}$ & $\begin{array}{l}\text { Employee } \\
\text { growth }\end{array}$ & $\begin{array}{l}\text { Asset } \\
\text { growth }\end{array}$ & $\begin{array}{l}\text { VC } \\
\text { funding }\end{array}$ & $\begin{array}{l}\text { going pub- } \\
\text { lic via IPO }\end{array}$ & $\begin{array}{l}\text { valuation of start- } \\
\text { up before VC } \\
\text { funding }\end{array}$ \\
\hline
\end{tabular}

\section{Firm controls}

\begin{tabular}{|c|c|c|c|c|c|}
\hline Firm age & ++++ & + & & & + \\
\hline Number of patents in possession & & & & & + \\
\hline Firm size (number of employees) & + & - & ++ & & + \\
\hline \multicolumn{6}{|l|}{ Team controls } \\
\hline ETMT size & ++ & & & & \\
\hline FT size & & + & & & \\
\hline Avg. age of founders & & & - & & \\
\hline $\begin{array}{l}\text { Avg. number of prior positions of } \\
\text { ETMT members }\end{array}$ & & & + & + & \\
\hline $\begin{array}{l}\text { Mean tenure of ETMT members } \\
\text { within the team }\end{array}$ & & & & + & \\
\hline $\begin{array}{l}\text { Tenure heterogeneity of ETMT } \\
\text { members within the team }\end{array}$ & & & & + & \\
\hline
\end{tabular}

\section{Industry controls}

\begin{tabular}{llll}
\hline Industry growth & + & + & \\
\hline Technological intensity & + & + & \\
\hline Competitive intensity & + & + \\
\hline Number of IPOs p.a. in industry & & + \\
\hline $\begin{array}{l}\text { Industry specific technological } \\
\text { change }\end{array}$ & & + \\
\hline Medical industry & & + \\
\hline Communications industry & & + \\
\hline Funding controls & & + \\
\hline External funding received & \\
\hline Angel investor prior to VC funding & & + \\
\hline Multiple funding offers received & & + \\
\hline Equity taken out after VC funding
\end{tabular}




\section{Acknowledgements}

We express our gratitude to the two anonymous reviewers who supported us in sharpening the focus of this paper.

\section{References}

Adler, Paul S. and Seok-Woo Kwon (2002): Social Capital: Prospects for a New Concept, Academy of Management Review, 27 (1): $17-40$.

Akgün, Ali E. and Gary S. Lynn (2002): Antecedents and Consequences of Team Stability on New Product Development Performance, Journal of Engineering \& Technology Management, 19 (3/4): 263-286.

Aldrich, Howard E. and Phillip H. Kim (2007): Small Worlds, Infinite Possibilities? How Social Networks Affect Entrepreneurial Team Formation and Search, Strategic Entrepreneurship Journal, 1 (1/2): 147-165.

Almus, Matthias and Eric Nerlinger (1999): Wachstumsdeterminanten junger innovativer Unternehmen: Empirische Ergebnisse für West-Deutschland, Jahrbücher für Nationalökonomie und Statistik, 218 (3/4): 257-275.

Amason, Allen C. (1996): Distinguishing the Effects of Functional and Dysfunctional Conflict on Strategic Decision Making: Resolving a Paradox for Top Management Teams, Academy of Management Journal, 39 (1): 123-148.

Amason, Allen C. and Harry J. Sapienza (1997): The Effects of Top Management Team Size and Interaction Norms on Cognitive and Affective Conflict, Journal of Management, 23 (4): 495-516.

Amason, Allen C., Rodney C. Shrader, and George H. Tompson (2006): Newness and Novelty: Relating Top Management Team Composition to New Venture Performance, Journal of Business Venturing, 21 (1): 125-148.

Barney, Jay (1991): Firm Resources and Sustained Competitive Advantage, Journal of Management, 17 (1): 99-120.

Baron, Robert A. (1988): Attributions and Organizational Conflict: The Mediating Role of Apparent Sincerity, Organizational Behavior \& Human Decision Processes, 41 (1): 111-127.

Baron, James N. and Michael T. Hannan (2002): Organizational Blueprints for Success in High-Tech Start-Ups: Lessons From the Stanford Project on Emerging Companies, California Management Review, 44 (3): 8-36.

Barringer, Bruce R., Foard F. Jones, and Donald O. Neubaum (2005): A Quantitative Content Analysis of the Characteristics of Rapid-Growth Firms and Their Founders, Journal of Business Venturing, 20 (5): 663-687.

Baum, Joel A. C. and Brian S. Silverman (2004): Picking Winners or Building Them? Alliance, Intellectual, and Human Capital as Selection Criteria in Venture Financing and Performance of Biotechnology Startups, Journal of Business Venturing, 19 (3): 411436.

Becker, Gary S. (1964): Human Capital: A Theoretical and Empirical Analysis, With Special Reference to Education, Columbia University Press: New York, NY et al.

Beckman, Christine M. and M. Diane Burton (2008): Founding the Future: Path Dependence in the Evolution of Top Manage- ment Teams From Founding to IPO, Organization Science, 19 (1): 3-24.

Beckman, Christine M., M. Diane Burton, and Charles O'Reilly (2007): Early Teams: The Impact of Team Demography on VC Financing and Going Public, Journal of Business Venturing, 22 (2): 147-173.

Block, Fred L. (1990): Post Industrial Possibilities: A Critique of Economic Discourse, University of California Press: Los Angeles, CA.

Boeker, Warren (1997): Executive Migration and Strategic Change: The Effect of Top Manager Movement on ProductMarket Entry, Administrative Science Quarterly, 42 (2): 213-236.

Boeker, Warren and Robert Wiltbank (2005): New Venture Evolution and Managerial Capabilities, Organization Science, 16 (2): 123-133

Bollen, Kenneth A. and Rick H. Hoyle (1990): Perceived Cohesion: A Conceptual and Empirical Examination, Social Forces, 69 (2): 479-504

Brettel, Malte (2002): Entscheidungskriterien von Venture Capitalists: Eine Empirische Analyse im internationalen Vergleich, Die Betriebswirtschaft, 62 (3): 305-325.

Burt, Ronald S. (1995): Structural Holes. The Social Structure of Competition, Harvard University Press: Cambridge, MA.

Busenitz, Lowell W., James O. Fiet, and Douglas D. Moesel (2004): Reconsidering the Venture Capitalists' "Value Added" Proposition: An Interorganizational Learning Perspective, Journal of Business Venturing, 19 (6): 787-807.

Busenitz, Lowell W., James O. Fiet, and Douglas D. Moesel (2005): Signaling in Venture Capitalist-New Venture Team Funding Decisions: Does it Indicate Long-Term Venture Outcomes?, Entrepreneurship: Theory \& Practice, 29 (1): 1-12.

Busenitz, Lowell W., Douglas D. Moesel, James O. Fiet, and Jay B. Barney (1997): The Framing of Perceptions of Fairness in the Relationship Between Venture Capitalists and New Venture Teams, Entrepreneurship: Theory \& Practice, 21 (3): 5-21.

Carlsson, Gosta and Katarina Karlsson (1970): Age, Cohorts and the Generation of Generations, American Sociological Review, 35 (4): 710-718.

Carpenter, Mason A., Marta A. Geletkanycz, and Wm. Gerard Sanders (2004): Upper Echelons Research Revisited: Antecedents, Elements, and Consequences of Top Management Team Composition, Journal of Management, 30 (6): 749-778.

Castanias, Richard P. and Constance E. Helfat (2001): The Managerial Rents Model: Theory and Empirical Analysis, Journal of Management, 27 (6): 661-678.

Chaganti, Rajeswararao S., Allison D. Watts, Radha Chaganti, and Monica Zimmerman-Treichel (2008): Ethnic-Immigrants in Founding Teams: Effects on Prospector Strategy and Performance in New Internet Ventures, Journal of Business Venturing, 23 (1): 113-139.

Chandler, Gaylen N., Benson Honig, and Johan Wiklund (2005): Antecedents, Moderators, and Performance Consequences of Membership Change in New Venture Teams, Journal of Business Venturing, 20 (5): 705-725.

Chlosta, Simone and Dorothea Kissel (2011): Franchise- vs. Neugründung: Analyse der Einflüsse auf Gründungsart und Gründungserfolg, Zeitschrift für Betriebswirtschaft, 81 (9): 945-975. 
Chowdhury, Sanjib (2005): Demographic Diversity for Building an Effective Entrepreneurial Team: Is it Important?, Journal of Business Venturing, 20 (6): 727-746.

Coleman, James S. (1988): Social Capital in the Creation of Human Capital, in: Christopher Winship and Sherwin Rosen (eds.): Organizations and Institutions: Sociological and Economic Approaches to the Analysis of Social Structure, American Journal of Sociology 94, Supplement; University of Chicago Press: Chicago, IL, 95-120.

Colombo, Massimo G. and Luca Grilli (2005): Founders' Human Capital and the Growth of New Technology-Based Firms: A Competence-Based View, Research Policy, 34 (6): 795-816.

Connelly, Brian L., S. Trevis Certo, R. Duane Ireland, and Christopher R. Reutzel (2011): Signaling Theory: A Review and Assessment, Journal of Management, 37 (1): 39-67.

Cooney, Thomas M. (2005): Editorial: What is an Entrepreneurial Team?, International Small Business Journal, 23 (3): 226-235.

Cooper, Arnold C. and Albert V. Bruno (1977): Success Among High-Technology Firms, Business Horizons, 20 (2): 16-22.

Cooper, Arnold C., F. Javier Gimeno-Gascon, and Carolyn Y. Woo (1994): Initial Human and Financial Capital as Predictors of New Venture Performance, Journal of Business Venturing, 9 (5): 371395 .

Cooper, Harris M. (1988): Organizing Knowledge Syntheses: A Taxonomy of Literature Reviews, Knowledge, Technology \& Policy, 1 (1): 104-126.

Cooper, Harris M. (1994): The Handbook of Research Synthesis, Sage: New York, NY.

Davila, Antonio and George Foster (2005): Management Accounting Systems Adoption Decisions: Evidence and Performance Implications From EarlySstage/Startup Companies, Accounting Review, 80 (4): 1039-1068.

Delarue, Anne, Geert van Hootegem, Stephen Procter, and Mark Burridge (2008): Teamworking and Organizational Performance: A Rreview of Survey-Based Research, International Journal of Management Reviews, 10 (2): 127-148.

Dess, Gregory G. and Jason D. Shaw (2001): Voluntary Turnover, Social Capital, and Organizational Performance, Academy of Management Review, 26 (3): 446-456.

Eisenhardt, Kathleen M. and Claudia Bird Schoonhoven (1990): Organizational Growth: Linking Founding Team, Strategy, Environment, and Growth Among U.S. Semiconductor Ventures, 1978-1988, Administrative Science Quarterly, 35 (3): 504-529.

Ennen, Edgar and Ansgar Richter (2010): The Whole Is More Than the Sum of Its Parts - or Is It? A Review of the Empirical Literature on Complementarities in Organizations, Journal of Management, 36 (1): 207-233.

Ensley, Michael D., James W. Carland, and JoAnn C. Carland (1998): The Effect of Entrepreneurial Team Skill Heterogeneity and Functional Diversity on New Venture Performance, Journal of Business and Entrepreneurship, 10 (1): 1-14.

Ensley, Michael D. and Keith M. Hmieleski (2005): A Comparative Study of New Venture Top Management Team Composition, Dynamics and Performance Between University-Based and Independent Start-Ups, Research Policy, 34 (7): 1091-1105.

Ensley, Michael D., Keith M. Hmieleski, and Craig L. Pearce (2006): The Importance of Vertical and Shared Leadership
Within New Venture Top Management Teams: Implications for the Performance of Startups, The Leadership Quarterly, 17 (3): 217-231.

Ensley, Michael D. and Craig L. Pearce (2001): Shared Cognition in Top Management Teams: Implications for New Venture Performance, Journal of Organizational Behavior, 22 (2): 145-160.

Ensley, Michael D., Craig L. Pearce, and Keith M. Hmieleski (2006): The Moderating Effect of Environmental Dynamism on the Relationship Between Entrepreneur Leadership Behavior and New Venture Performance, Journal of Business Venturing, 21 (2): 243-263.

Ensley, Michael D. and Allison W. Pearson (2005): An Exploratory Comparison of the Behavioral Dynamics of Top Management Teams in Family and Nonfamily New Ventures: Cohesion, Conflict, Potency, and Consensus, Entrepreneurship: Theory \& Practice, 29 (3): 267-284.

Ensley, Michael D., Allison W. Pearson, and Allen C. Amason (2002): Understanding the Dynamics of New Venture Top Management Teams: Cohesion, Conflict, and New Venture Performance, Journal of Business Venturing, 17 (4): 365-386.

Ensley, Michael D., Allison W. Pearson, and Craig L. Pearce (2003): Top Management Team Process, Shared Leadership, and New Venture Performance: A Theoretical Model and Research Agenda, Human Resource Management Review, 13 (2): 329-346.

Erikson, Truls (2002): Entrepreneurial Capital: The Emerging Venture's Most Important Asset and Competitive Advantage, Journal of Business Venturing, 17 (3): 275-290.

Finkelstein, Sydney (1992): Power in Top Management Teams: Dimensions, Measurement, and Validation, Academy of Management Journal, 35 (3): 505-538.

Finkelstein, Sydney, Donald C. Hambrick, and Albert A. Cannella (2009): Strategic Leadership: Theory and Research on Executives, Top Management Teams, and Boards, Oxford University Press: New York, NY.

Franke, Nikolaus, Marc Gruber, Dietmar Harhoff, and Joachim Henkel (2008): Venture Capitalists' Evaluations of Start-Up Teams: Trade-Offs, Knock-Out Criteria, and the Impact of VC Experience, Entrepreneurship: Theory \& Practice, 32 (3): 459483 .

Franke, Nikolaus, Marc Gruber, Joachim Henkel, and Karin Hoisl (2004): Die Bewertung von Gründerteams durch VentureCapital-Geber: Eine empirische Analyse, Die Betriebswirtschaft, 64 (6): 651-670.

Gartner, William B. (1990): What Are We Talking About When We Talk About Entrepreneurship?, Journal of Business Venturing, 5 (1): 15-28.

Gebert, Diether, Sabine Boerner, and Ralf Lanwehr (2003): The Risks of Autonomy: Empirical Evidence for the Necessity of a Balance Management in Promoting Organizational Innovativeness, Creativity \& Innovation Management, 12 (1): 41-49.

Gerber, Michael E. (1995): The E-Myth Revisited: Why Most Small Businesses Don't Work and What To Do About It, HarperBusiness: New York, NY.

Gimeno, Javier, Timothy B. Folta, Arnold C. Cooper, and Carolyn Y. Woo (1997): Survival of the Fittest? Entrepreneurial Human Capital and the Persistence of Underperforming Firms, Administrative Science Quarterly, 42 (4): 750-783. 
Hambrick, Donald C. and Phyllis A. Mason (1984): Upper Echelons: The Organization as a Reflection of Its Top Managers, Academy of Management Review, 9 (2): 193-206.

Harris, Dawn and Constance Helfat (1997): Specificity of CEO Human Capital and Compensation, Strategic Management Journal, 18 (11): 895-920.

Harrison, David A., Kenneth H. Price, and Myrtle P. Bell (1998): Beyond Relational Demography: Time and the Effects of Surfaceand Deep-Level Diversity on Work Group Cohesion, Academy of Management Journal, 41 (1): 96-107.

Hayes, Rachel M., Paul Oyer, and Scott Schaefer (2004): CoWorker Complementarity and the Stability of Top Management Teams, Working Paper, http://ideas.repec.org/p/nbr/nberwo/ 10350.html (Access date: 2010-05-10).

Heinen, J. Stephen and Eugene Jacobson (1976): A Model of Task Group Development in Complex Organizations and a Strategy of Implementation, Academy of Management Review, 1 (4): 98-111.

Hellmann, Thomas and Manju Puri (2002): Venture Capital and the Professionalization of Start-Up Firms: Empirical Evidence, Journal of Finance, 57 (1): 169-197.

Hmieleski, Keith M. and Michael D. Ensley (2007): A Contextual Examination of New Venture Performance: Entrepreneur Leadership Behavior, Top Management Team Heterogeneity, and Environmental Dynamism, Journal of Organizational Behavior, 28 (7): 865-889.

Hornaday, Robert W. (1992): Thinking About Entrepreneurship: A Fuzzy Set Approach, Journal of Small Business Management, $30(4): 12-23$

Hsu, David H. (2007): Experienced Entrepreneurial Founders, Organizational Capital, and Venture Capital Funding, Research Policy, 36 (5): 722-741.

Hvide, Hans K. and Jarle Moen (2010): Lean and Hungry or Fat and Content? Entrepreneurs' Wealth and Start-Up Performance, Management Science, 56 (8): 1242-1258.

Jackson, Susan E. (1992): Consequences of Group Composition for the Interpersonal Dynamics of Strategic Issue Processing, in: Paul Shrivastava, Anne Huff, and Jane Dutton (eds.): Advances in Strategic Management: A Research Annual, JAI Press: Greenwich, CT and London, 345-382.

Jehn, Karen A. (1995): A Multimethod Examination of the Benefits and Detriments of Intragroup Conflict, Administrative Science Quarterly, 40 (2): 256-282.

Kamm, Judith B. and Aaron J. Nurick (1993): The stages of Team Venture Formation: A Decision-Making Model, Entrepreneurship: Theory \& Practice, 17 (2): 17-27.

Kamm, Judith B., Jeffrey C. Shuman, John A. Seeger, and Aaron J. Nurick (1990): Entrepreneurial Teams in New Venture Creation: A Research Agenda, Entrepreneurship: Theory \& Practice, 14 (4): 7-17.

Kaplan, Steven N. and Per Strömberg (2004): Characteristics, Contracts, and Actions: Evidence From Venture Capitalist Analyses, Journal of Finance, 59 (5): 2177-2210.

Kemery, Edward R., Arthur G. Bedeian, Kevin W. Mossholder, and John Touliatos (1985): Outcomes of Role Stress: A Multisample Constructive Replication, Academy of Management Journal, 28 (2): 363-375.
Kor, Yasemin Y. (2003): Experience-Based Top Management Team Competence and Sustained Growth, Organization Science, 14 (6): 707-719.

Kor, Yasemin Y. and Joseph T. Mahoney (2000): Penrose's Resource-Based Approach: The Process and Product of Research Creativity, Journal of Management Studies, 37 (1): 109-139.

Liu, Amy Qiaoming and Terry Besser (2003): Social Capital and Participation in Community Improvement Activities by Elderly Residents in Small Towns and Rural Communities, Rural Sociology, 68 (3): 343-365.

McEvoy, Glenn M. and Wayne F. Cascio (1987): Do Good or Poor Performers Leave? A Meta-Analysis of the Relationship Between Performance and Turnover, Academy of Management Journal, 30 (4): 744-762.

McGee, Jeffrey E. and Michael J. Dowling (1994): Using R\&D Cooperative Arrangements to Leverage Managerial Experience: A Study of Technology-Intensive New Ventures, Journal of Business Venturing, 9 (1): 33-48.

McGee, Jeffrey E., Michael J. Dowling, and William L. Megginson (1995): Cooperative Strategy and New Venture Performance: The Role of Business Strategy and Management Experience, Strategic Management Journal, 16 (7): 565-580.

Meyer, Alan D., Anne S. Tsui, and C. R. Hinings (1993): Configurational Approaches to Organizational Analysis, Academy of Management Journal, 36 (6): 1175-1195.

Miller, C. Chet, Linda M. Burke, and William H. Glick (1998): Cognitive Diversity Among Upper-Echelon Executives: Implications for Strategic Decision Processes, Strategic Management Journal, 19 (1): 39-58.

Murray, Alan I. (1989): Top Management Group Heterogeneity and Firm Performance, Strategic Management Journal, 10 (Special Issue): 125-141.

Newbert, Scott L. (2007): Empirical Research on the ResourceBased View of the Firm: An Assessment and Suggestions for Future Research, Strategic Management Journal, 28 (2): 121146.

O'Reilly III, Charles A., David F. Caldwell, and William P. Barnett (1989): Work Group Demography, Social Integration, and Turnover, Administrative Science Quarterly, 34 (1): 21-37.

Packalen, Kelley A. (2007): Complementing Capital: The Role of Status, Demographic Features, and Social Capital in Founding Teams' Abilities to Obtain Resources, Entrepreneurship: Theory \& Practice, 31 (6): 873-891.

Parise, Salvatore and Keith Rollag (2010): Emergent Network Structure and Initial Group Performance: The Moderating Role of Pre-Existing Relationships, Journal of Organizational Behavior, 31 (6): 877-897.

Patzelt, Holger, Dodo zu Knyphausen-Aufseß, and Petra Nikol (2008): Top Management Teams, Business Models, and Performance of Biotechnology Ventures: An Upper Echelon Perspective, British Journal of Management, 19 (3): 205-221.

Pearce, Craig L., Henry P. Sims, Jonathan F. Cox, Gail Ball, Eugene Schnell, Ken A. Smith, and Linda Trevino (2003): Transactors, Transformers and Beyond, Journal of Management Development, 22 (4): 273-307. 
Pelled, Lisa H. (1996): Demographic Diversity, Conflict, and Work Group Outcomes: An Intervening Process Theory, Organization Science, 7 (6): 615-631.

Penrose, Edith T. (1959): The Theory of the Growth of the Firm, Blackwell: Oxford.

Putnam, Robert D., Robert Leonardi, and Raffaella Y. Nanetti (1994): Making Democracy Work: Civic Traditions in Modern Italy, $5^{\text {th }}$ ed., Princeton University Press: Princeton, NJ.

Rafferty, Alannah E. and Mark A. Griffin (2004): Dimensions of Transformational Leadership: Conceptual and Empirical Extensions, Leadership Quarterly, 15 (3): 329-354.

Randolph, Justus J. (2009): A Guide to Writing the Dissertation Literature Review, Practical Assessment, Research \& Evaluation, 14 (13): $1-13$.

Rubenson, George C. and Anil K. Gupta (1996): The Initial Succession: A Contingency Model of Founder Tenure, Entrepreneurship: Theory \& Practice, 21 (2): 21-35.

Sahaym, Arvin (2005): Spin-Out Formation and Performance From the Lens of Dynamic Capabilities and Social Capital Theory, Academy of Management Annual Meeting Proceedings: M1-5.

Salancik, Gerald R. and Jeffrey Pfeffer (1978): A Social Information Processing Approach to Job Attitudes and Task Design, Administrative Science Quarterly, 23 (2): 224-253.

Sapienza, Harry J. and Curtis M. Grimm (1997): Founder Characteristics, Start-Up Process, and Strategy/Structure Variables as Predictors of Shortline Railroad Performance, Entrepreneurship: Theory \& Practice, 22 (1): 5-24.

Schmelter, Anja (2004): Entwicklungsverläufe forschungsnaher Unternehmensgründungen und deren Determinanten, Die Betriebswirtschaft, 64 (4): 471-486.

Schrör, Hartmut (2007): Unternehmensdemografie: Zunahme des Unternehmensbestands, http://epp.eurostat.ec.europa.eu/ cache/ITY_OFFPUB/KS-SF-07-048/DE/KS-SF-07-048-DE.PDF (Access date: 2012-05-10).

Shane, Scott and Toby Stuart (2002): Organizational Endowments and the Performance of University Start-Ups, Management Science, 48 (1): 154-170.

Shrader, Rod and Donald S. Siegel (2007): Assessing the Relationship Between Human Capital and Firm Performance: Evidence From Technology-Based New Ventures, Entrepreneurship: Theory \& Practice, 31 (6): 893-908.

Simons, Tony, Lisa H. Pelled, and Ken A. Smith (1999): Making Use of Difference: Diversity, Debate, and Decision Comprehensiveness in Top Management Teams, Academy of Management Journal, 42 (6): 662-673

Smith, Ken G., Ken A. Smith, Henry P. Sims, Douglas P. O'Bannon, Judith A. Scully, and Judy D. Olian (1994): Top Management Team Demography and Process: The Role of Social Integration and Communication, Administrative Science Quarterly, 39 (3): 412-438.

Spence, Michael (1973): Job Market Signaling, Quarterly Journal of Economics, 87 (3): 355-374.
Spreitzer, Gretchen M. (1996): Social Structural Characteristics of Psychological Empowerment, Academy of Management Journal, 39 (2): 483-504.

Stinchcombe, Arthur L. (1965): Social Structure and Organizations, in: James G. March (ed.): Handbook of organizations, Rand: Chicago, IL, 142-193.

Stuart, Robert W. and Pier A. Abetti (1990): Impact of Entrepreneurial and Management Experience on Early Performance, Journal of Business Venturing, 5 (3): 151-162.

Sweetland, Scott R. (1996): Human Capital Theory: Foundations of a Field of Inquiry, Review of Educational Research, 66 (3): 341-359.

Tacheva, Sabina (2007): Top Management Team Diversity: A Multilevel Exploration of Antecedents and Consequences, Dissertation, Graduate School of Business Administration, Economics, Law and Social Sciences (HSG), University of St. Gallen.

Ucbasaran, Deniz, Andy Lockett, Mike Wright, and Paul Westhead (2003): Entrepreneurial Founder Teams: Factors Associated With Member Entry and Exit, Entrepreneurship: Theory \& Practice, 28 (2): 107-127.

Vanaelst, Iris, Bart Clarysse, Mike Wright, Andy Lockett, Nathalie Moray, and Rosette S'Jegers (2006): Entrepreneurial Team Development in Academic Spinouts: An Examination of Team Heterogeneity, Entrepreneurship: Theory \& Practice, 30 (2): 249-271.

Vissa, Balagopal and Aya S. Chacar (2009): Leveraging Ties: The Contingent Value of Entrepreneurial Teams' External Advice Networks on Indian Software Venture Performance, Strategic Management Journal, 30 (11): 1179-1191.

Weinzimmer, Laurence G. (1997): Top Management Team Correlates of Organizational Growth in a Small Business Context: A Comparative Study, Journal of Small Business Management, 35 (3): 1-10.

Wernerfelt, Birger (1984): A Resource-Based View of the Firm, Strategic Management Journal, 5 (2): 171-180.

West, G. Page (2007): Collective Cognition: When Entrepreneurial Teams, Not Individuals, Make Decisions, Entrepreneurship: Theory \& Practice, 31 (1): 77-102.

Wiersema, Margarethe F. and Karen A. Bantel (1992): Top Management Team Demography and Corporate Strategic Change, Academy of Management Journal, 35 (1): 91-121.

Witt, Peter (2004): Entrepreneurs' Networks and the Success of Start-Ups, Entrepreneurship and Regional Development, 16 (5): 391-412.

Woolcock, Michael and Deepa Narayan (2000): Social Capital: Implications for Development Theory, Research, and Policy, World Bank Research Observer, 15 (2): 225-249.

Zimmerman, Monica A. (2008): The Influence of Top Management Team Heterogeneity on the Capital Raised Through an Initial Public Offering, Entrepreneurship: Theory \& Practice, 32 (3): 391-414. 


\section{Biographies}

Konstantin Maschke is a doctoral candidate at the Department of Strategic Leadership and Global Management at the Technical University Berlin, Germany. He received his diploma in Business Information Technology at the University of Mannheim, Germany, in spring 2007. His research interests include entrepreneurship, strategic management, team compositions and group dynamics.
Dodo zu Knyphausen-Aufse $\boldsymbol{B}$ is Professor of Strategic Leadership and Global Management at the Technical University Berlin, Germany. His work is located at the intersection of strategic management and entrepreneurship. Moreover, he is interested in the impact of external stakeholders on the strategy development of business firms. 